\title{
Microscopic theory of intrinsic timescales in spiking neural networks
}

\author{
Alexander van Meegen $\odot^{*}$ and Sacha J. van Albada $\odot$ \\ Institute of Neuroscience and Medicine (INM-6) and Institute for Advanced Simulation (IAS-6) and JARA-Institut \\ Brain Structure-Function Relationships (INM-10), Jülich Research Centre, 52425 Jülich, Germany \\ and Institute of Zoology, University of Cologne, 50674 Cologne, Germany
}

(Received 2 July 2021; accepted 21 September 2021; published 28 October 2021)

\begin{abstract}
A complex interplay of single-neuron properties and the recurrent network structure shapes the activity of cortical neurons. The single-neuron activity statistics differ in general from the respective population statistics, including spectra and, correspondingly, autocorrelation times. We develop a theory for self-consistent secondorder single-neuron statistics in block-structured sparse random networks of spiking neurons. In particular, the theory predicts the neuron-level autocorrelation times, also known as intrinsic timescales, of the neuronal activity. The theory is based on an extension of dynamic mean-field theory from rate networks to spiking networks, which is validated via simulations. It accounts for both static variability, e.g., due to a distributed number of incoming synapses per neuron, and temporal fluctuations of the input. We apply the theory to balanced random networks of generalized linear model neurons, balanced random networks of leaky integrate-and-fire neurons, and a biologically constrained network of leaky integrate-and-fire neurons. For the generalized linear model network with an error function nonlinearity, a novel analytical solution of the colored noise problem allows us to obtain self-consistent firing rate distributions, single-neuron power spectra, and intrinsic timescales. For the leaky integrate-and-fire networks, we derive an approximate analytical solution of the colored noise problem, based on the Stratonovich approximation of the Wiener-Rice series and a novel analytical solution for the free upcrossing statistics. Again closing the system self-consistently, in the fluctuation-driven regime, this approximation yields reliable estimates of the mean firing rate and its variance across neurons, the interspike-interval distribution, the single-neuron power spectra, and intrinsic timescales. With the help of our theory, we find parameter regimes where the intrinsic timescale significantly exceeds the membrane time constant, which indicates the influence of the recurrent dynamics. Although the resulting intrinsic timescales are on the same order for generalized linear model neurons and leaky integrate-and-fire neurons, the two systems differ fundamentally: for the former, the longer intrinsic timescale arises from an increased firing probability after a spike; for the latter, it is a consequence of a prolonged effective refractory period with a decreased firing probability. Furthermore, the intrinsic timescale attains a maximum at a critical synaptic strength for generalized linear model networks, in contrast to the minimum found for leaky integrate-and-fire networks.
\end{abstract}

DOI: 10.1103/PhysRevResearch.3.043077

\section{INTRODUCTION}

Neural dynamics in the cerebral cortex of awake behaving animals unfolds over multiple timescales, ranging from milliseconds up to seconds and more [1-5]. Such a heterogeneity of timescales in the dynamics is a substrate for temporal processing of sensory stimuli [6] and reflects integration of information over different time intervals [3,4]. Intriguingly, in vivo electrophysiological recordings reveal a structure in the autocorrelation timescales of the activity on the level of single neurons $[2,7]$. This structure could arise from systematic variations in single-neuron or synaptic properties [8,9], from the intricate cortical network structure [10], or from a

*avm@physik.hu-berlin.de

Published by the American Physical Society under the terms of the Creative Commons Attribution 4.0 International license. Further distribution of this work must maintain attribution to the author(s) and the published article's title, journal citation, and DOI. combination of both $[11,12]$. Furthermore, timescales may be influenced by the external input to the network, and depend on the chosen measurement procedure [13]. Thus, while these timescales are referred to as intrinsic timescales, they are shaped by intrinsic and extrinsic factors alike.

Explaining the timescales of individual neurons embedded in a network poses a theoretical challenge: How to account for a microscopic, neuron-level observable in a macroscopic theory? Clearly, a straightforward coarse-graining of the activity eliminates the microscopic observable of interest [14]. Dynamic mean-field theory (DMFT) [15-17] makes microscopic observables accessible because, instead of coarse-graining the activity of the neurons, it coarse-grains their input. Here, the term "dynamic" specifies that the input is approximated as a stochastic process that varies in time, in contrast to the notion of a mean-field theory in physics, which usually describes processes embedded in a constant field. DMFT has led to significant insights into the interrelation between network structure and intrinsic timescales for recurrent networks of (nonspiking) rate neurons [15-23]. In particular, it has been shown that very slow intrinsic timescales emerge close to 
a transition to chaos in autonomous networks [15]. Interestingly, simply adding a noisy input to the network significantly reduces this effect and even leads to a novel dynamical regime [21]. Furthermore, increasing the complexity of the singleneuron dynamics reveals that timescales of slow adaptive currents are not straightforwardly expressed in the network dynamics [22], and leads to yet another dynamical regime termed "resonant chaos" [23]. In combination, these results suggest that the mechanisms shaping the intrinsic timescales in recurrent networks are highly involved.

A characteristic feature of neural communication in the brain is the spike-based coupling [24]: the output of a neuron is a stereotypical pulse, a spike, that is produced once the internal voltage exceeds a threshold and that travels along the axon to the target neurons. Consequently, spiking neural network models have already yielded notable insights into cortical neural dynamics. Prominent examples are the excitatory-inhibitory balance mechanism which dynamically generates strong fluctuations while keeping the activity in a physiological range $[25,26]$ and the mechanism of recurrent inhibitory feedback leading to low cross-correlation between neurons despite the high number of shared inputs [27,28]. From a theoretical perspective, spike-based coupling further increases the complexity of the dynamics. This calls for an extension of DMFT to spiking networks. Following early works where slow synaptic dynamics reduced the spiking networks effectively to rate networks [18,29], this was recently achieved with a model-independent framework [30] (see also the pioneering work [31]).

Perhaps unintuitively, the main obstacle is not the reduction of the recurrent dynamics to the DMFT but the colored noise problem: to obtain the output statistics of the neuron for temporally correlated input statistics. Previous works relied on numerical methods to address the colored noise problem [31-36] because the spiking nonlinearity renders this problem in general analytically intractable. Such a self-consistent numerical scheme already revealed an unexpected minimum instead of a maximum in the intrinsic timescales for spiking networks at a critical coupling strength [37]. However, numerical solutions have the drawback that they lead to noisy estimates of the autocorrelation function, which poses additional challenges on the inference of intrinsic timescales [38] and other dynamical quantities from the neuronal and network parameters. In addition, such a self-consistent numerical scheme is computationally intensive.

In this paper, we use analytical approaches to close the selfconsistency equations for spiking networks. First, we transfer the theory for rate networks to one for spiking networks starting from the characteristic functional of the recurrent input. This shows that the first two cumulants (mean and variance) of the connectivity matrix suffice to fully characterize the effective stochastic input, and automatically take the static variabilities (firing rate, indegree) in the network into account. Since it is based on DMFT, the resulting theory indeed accounts for the timescales on the microscopic level, orthogonal to approaches where the activity of a population of neurons is reduced to an effective mesoscopic description [39]. Second, we derive an analytical solution to the colored noise problem for generalized linear model (GLM) neurons with exponential and error function nonlinearity. Using these analytical solu- tions, we validate that the self-consistent DMFT captures both the static second-order statistics, the distribution of firing rates across neurons, and the dynamic second-order statistics, the population-averaged autocorrelation function. Furthermore, we use the theory to investigate the conditions for longer intrinsic timescales, like those observed in in vivo electrophysiological recordings [2,7], in a balanced random network of GLM neurons. Due to the analytical tractability, our theory exposes the factors that shape the intrinsic timescale. Third, we derive a numerically efficient analytical approximation for the colored noise problem for leaky integrate-and-fire (LIF) neurons in the noise-driven regime based on the Wiener-Rice series and the Stratonovich approximation thereof [40,41]. For a different approach based on a Markovian embedding, which leads to multidimensional Fokker-Planck equations with involved boundary conditions that are solved numerically, see [42]. In contrast, our approximation leads to integrals of which the computationally most involved ones can be solved analytically. Lastly, we use these results to explore the parameter space of a balanced random network of LIF neurons for long timescales, and apply the theory to a more elaborate model with population-specific connection probabilities that are constrained by biological data [43].

We start this manuscript with the derivation of the DMFT equations from the characteristic functional of the recurrent input. The remainder of the results is structured according to the neuron model. First we consider GLM neurons with exponential and error function nonlinearity, respectively, then we turn to LIF neurons. For each neuron model, we begin by deriving the solution or approximation of the colored noise problem. We then describe the numerical method to solve the self-consistent DMFT equations for the given neuron type (GLM or LIF). Subsequently, we use our theory to investigate the timescale in the respective network models.

\section{MICROSCOPIC THEORY OF INTRINSIC TIMESCALES}

We consider random network topologies where the entries of the matrix $\mathcal{J}$ containing the synaptic strengths, i.e., the amplitudes of evoked post-synaptic currents due to incoming spikes, are independent and identically distributed (i.i.d.). A synapse from neuron $j$ to neuron $i$ exists ( $\mathcal{J}_{i j}$ is nonzero) with probability $p$; each nonzero entry $J_{i j}$ is independently sampled from the distribution of synaptic strengths with mean $\mu_{J}$ and variance $\sigma_{J}^{2}<\infty$ :

$$
\mathcal{J}_{i j}=\left\{\begin{array}{ll}
J_{i j} & \text { with probability } p \\
0 & \text { with probability } 1-p
\end{array} .\right.
$$

The connectivity is thus taken to be pairwise Bernoulli, yielding maximally one synapse from a given presynaptic to a given postsynaptic neuron. To account for Dale's law and further heterogeneities, we subdivide the network into populations, e.g., all pyramidal cells in cortical layer V, consisting of statistically identical neurons and denote the population by a Greek superscript. Within this generalization, the entries of $\mathcal{J}$ are still i.i.d. random numbers for a given pair of populations $\alpha, \beta$, but $p^{\alpha \beta}$ and the distribution of $J_{i j}^{\alpha \beta}$ can vary for different pairs of populations [Fig. 1(a)]. For example, if $I$ denotes a population of inhibitory interneurons, all $J_{i j}^{\alpha I}$ are negative. 
(a)

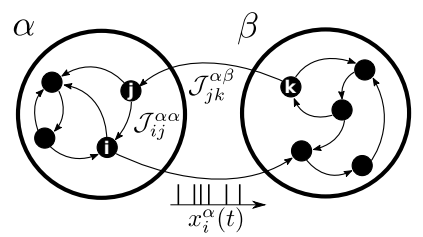

(c)

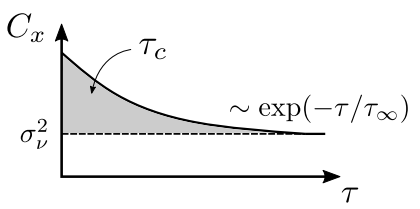

(b)

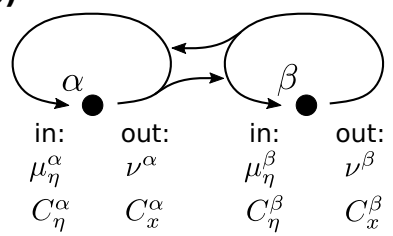

(d)

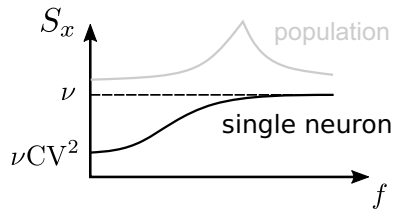

FIG. 1. Illustration of the theory. (a) We consider populations of randomly connected neurons $(\alpha, \beta)$ that communicate via spike trains $x_{i}^{\alpha}(t)$. The neurons of population $\beta$ are connected to those of population $\alpha$ with connection probability $p^{\alpha \beta}$. (b) The theory reduces a population to a single neuron driven by an effective stochastic input $\eta^{\alpha}$. The first- and second-order statistics $\mu_{\eta}^{\alpha}$ and $C_{\eta}^{\alpha}$ of $\eta^{\alpha}$ depend self-consistently on the output statistics, $\nu^{\alpha}$ and $C_{x}^{\alpha}$. (c) From the stationary spike train autocorrelation function $C_{x}(\tau)=v \delta(\tau)+\hat{C}_{x}(\tau)$, we obtain the correlation time $\tau_{c}$, the asymptotic decay $\tau_{\infty}$, and the variability of the rate across neurons, $\sigma_{v}^{2}$. (d) Instead of the stationary autocorrelation function we sometimes consider the power spectrum $S_{x}(f)$, which saturates at the firing rate, $S_{x}(f) \stackrel{f \rightarrow \infty}{\rightarrow} v$, and, for a renewal process, has the zero-frequency limit $S_{x}(f) \stackrel{f \rightarrow 0}{\rightarrow} \nu \mathrm{CV}^{2}$. Throughout, we consider the population-averaged single-unit statistics (black curve) instead of the statistics of the population-averaged activity (gray curve).

In this manuscript, we focus on the situation where the average number of synapses per neuron, the indegree $K^{\alpha \beta}=$ $p^{\alpha \beta} N^{\beta}$, is large: $K^{\alpha \beta} \gg 1$ due to a large number of presynaptic neurons $N^{\beta} \gg 1$ in combination with a moderate connection probability $p^{\alpha \beta}$ on the order of $10 \%$, in agreement with the situation in cortical networks [44]. In line with the theory of balanced networks [45], we assume that neither single spikes are sufficient to cause firing nor coherent input from all presynaptic neurons is necessary. Moreover, we consider networks which are in an asynchronous irregular state exhibited by cortical networks of awake, behaving animals [46].

In the following, we first consider a single population for clarity because the generalization to multiple populations is straightforward.

\section{A. Input statistics}

Dynamic mean-field theory reduces the dynamics of the recurrent network to a set of self-consistent stochastic equations. Its core idea is to approximate the recurrent input

$$
\eta_{i}(t)=\sum_{j=1}^{N} \mathcal{J}_{i j} x_{j}(t)
$$

by independent Gaussian processes. In Eq. (2), and throughout this manuscript, $x_{j}(t)=\sum_{n} \delta\left(t-t_{j, n}\right)$ denotes the spikes emitted at times $t_{j, n}$ by neuron $j$-the spike train of neuron $j$ - which are the output of neuron $j$ and contribute to the input of target neuron $i$. The sum in Eq. (2) extends over all $N$ neurons, using that $\mathcal{J}_{i j}=0$ for neurons that are not connected.

\section{Gaussian process approximation}

Here, we sketch the derivation to expose necessary conditions for the DMFT. For the full treatment of the problem, we refer to the model-independent DMFT developed in Ref. [30], which is applicable to spiking networks.

We start from the deterministic input Eq. (2) and derive its approximation as independent Gaussian processes. To this end, let us consider the characteristic functional of the recurrent input. Because $\eta_{i}(t)$ is a deterministic quantity, its distribution is a Dirac delta and its characteristic functional, defined by $\Phi_{\eta}[\boldsymbol{u}(t)]=\left\langle\exp \left(i \int_{0}^{T} \boldsymbol{u}(t)^{\top} \boldsymbol{\eta}(t) d t\right)\right\rangle_{\eta}$, is [40,47] (see also Appendix A, Eq. (A2))

$$
\Phi_{\eta}[\boldsymbol{u}(t)]=\exp \left(i \int_{0}^{T} \sum_{i, j=1}^{N} u_{i}(t) \mathcal{J}_{i j} x_{j}(t) d t\right) .
$$

In Eq. (3), $u_{i}(t)$ are arbitrary test functions; the derivatives of $\Phi_{\eta}[\boldsymbol{u}(t)]$ with respect to the test functions evaluated at $u_{i}(t)=$ 0 yield the moments of the recurrent input.

Now we assume that the dynamics of the system are, on a statistical level, very similar for any given realization of the connectivity, i.e., we assume that the system is self-averaging. Thus we can consider the average across realizations of $\mathcal{J}$ and neglect the dependence of the spike trains on the realization of $\mathcal{J}$ for this average. For the latter assumption, it is important to keep in mind that we consider the statistics of the entire network: while the spike train of a particular neuron is certainly highly correlated to the realization of the connectivity, self-averaging means that this does not hold for the statistics of the activity across the network. Put differently, the input to the neuron, and hence the neuron itself, "loses its identity" and becomes a statistical representative for an arbitrary neuron in the network.

Under these assumptions, the average of the characteristic functional is

$$
\begin{aligned}
\left\langle\Phi_{\eta}[\boldsymbol{u}(t)]\right\rangle_{\mathcal{J}} \approx & e^{i\langle\mathcal{J}\rangle \sum_{i, j=1}^{N} \int_{0}^{T} u_{i}(t) x_{j}(t) d t} \\
& \times e^{-\frac{1}{2}\left\langle\Delta \mathcal{J}^{2}\right\rangle \sum_{i, j=1}^{N}\left(\int_{0}^{T} u_{i}(t) x_{j}(t) d t\right)^{2}},
\end{aligned}
$$

where we used the independence of the $\mathcal{J}_{i j}$, their characteristic function $\left\langle\exp \left(i k_{i j} \mathcal{J}_{i j}\right)\right\rangle_{\mathcal{J}_{i j}}=\exp \left(i\langle\mathcal{J}\rangle k_{i j}-\frac{1}{2}\left\langle\Delta \mathcal{J}^{2}\right\rangle k_{i j}^{2}+\right.$ ...), and neglected the cumulants of $\mathcal{J}_{i j}$ beyond the second-order cumulant (the variance) $\left\langle\Delta \mathcal{J}^{2}\right\rangle$. Due to the independence of the $\mathcal{J}_{i j}$, the expectation factorizes into a product $\prod_{i, j=1}^{N}$ which leads to the sum $\sum_{i, j=1}^{N}$ in the exponent. Within each factor, the first (second) cumulant leads to a linear (quadratic) term in the exponent. Next, we rewrite the square, $\left(\int_{0}^{T} u_{i}(t) x_{j}(t) d t\right)^{2}=$ $\int_{0}^{T} \int_{0}^{T} u_{i}(t) u_{i}\left(t^{\prime}\right) x_{j}(t) x_{j}\left(t^{\prime}\right) d t d t^{\prime}$, and introduce the networkaveraged auxiliary fields

$$
\begin{gathered}
\mu_{\eta}(t)=\langle\mathcal{J}\rangle \sum_{j=1}^{N} x_{j}(t), \\
C_{\eta}\left(t, t^{\prime}\right)=\left\langle\Delta \mathcal{J}^{2}\right\rangle \sum_{j=1}^{N} x_{j}(t) x_{j}\left(t^{\prime}\right) .
\end{gathered}
$$


Using the auxiliary fields, the characteristic functional factorizes, $\left\langle\Phi_{\eta}[\boldsymbol{u}(t)]\right\rangle_{\mathcal{J}} \approx \prod_{i=1}^{N} \hat{\Phi}_{\eta}\left[u_{i}(t)\right]$, with the individual factors given by

$$
\hat{\Phi}_{\eta}[u(t)]=e^{i \int_{0}^{T} u(t) \mu_{\eta}(t) d t-\frac{1}{2} \int_{0}^{T} \int_{0}^{T} u(t) C_{\eta}\left(t, t^{\prime}\right) u\left(t^{\prime}\right) d t d t^{\prime}},
$$

which is the characteristic functional of a Gaussian process with mean $\mu_{\eta}(t)$ and correlation function $C_{\eta}\left(t, t^{\prime}\right)$ [40,47] (see Appendix A, Eq. (A3)). The factorization $\left\langle\Phi_{\eta}[\boldsymbol{u}(t)]\right\rangle_{\mathcal{J}} \approx$ $\prod_{i=1}^{N} \hat{\Phi}_{\eta}\left[u_{i}(t)\right]$ implies that the approximate inputs described by $\hat{\Phi}_{\eta}[u(t)]$ are independent across neurons.

The above sketch of a derivation reveals multiple assumptions we make in the DMFT. First, we assumed self-averaging. This is a necessary assumption if one wants to derive a statement that generalizes beyond a given connectivity matrix to its statistics only. For a broad class of rate networks, one can show rigorously that the statistics of the activity across the network are indeed self-averaging by calculating the distribution of the empirical measure $\frac{1}{N} \sum_{i=1}^{N} \delta\left[y(t)-x_{i}(t)\right]$ across realizations of the connectivity $[48,49]$. Here, we check this assumption post-hoc by comparison of the theory with simulations for a single realization of the connectivity. Second, we implicitly assumed $\bar{g}:=N\langle\mathcal{J}\rangle$ and $g^{2}:=N\left\langle\Delta \mathcal{J}^{2}\right\rangle$ do not scale with $N$ such that the auxiliary fields remain finite for large networks. Using the mean number of inputs per neuron $K=p N$ and the properties of $\mathcal{J}$, we get

$$
\bar{g}=K \mu_{J}, \quad g^{2}=K\left(\sigma_{J}^{2}+(1-p) \mu_{J}^{2}\right) .
$$

Third, we neglected higher cumulants of the input. Using the assumption $J_{i j}=O(1 / \sqrt{K})$ leads to $\mu_{J}=O(1 / \sqrt{K}), \sigma_{J}^{2}=$ $O(1 / K)$ and thus $\bar{g}=O(\sqrt{K}), g^{2}=O(1)$ as well as $O(1 / \sqrt{K})$ for the neglected higher cumulants. Accordingly, in the regime $K \gg 1$, neglecting the contributions from higher cumulants, e.g., due to shot noise effects [35], is justified.

\section{Self-consistency problem}

Given these assumptions, the recurrent inputs $\eta_{i}(t)$ can be approximated by independent Gaussian processes, which leads to a coarse-grained description of the dynamics: since all inputs are statistically equivalent, the neurons become statistically equivalent as well and the system reduces to $N$ independent, identical stochastic equations. For $N \gg 1$, we can replace the empirical averages in Eqs. (4) and (5) by ensemble averages such that we arrive at a set of self-consistency equations. This step can be made rigorous using the formalism of Ref. [30], see Eqs. (2) and (3) and Appendix 1 therein.

In the stationary state, the self-consistency equations are given by

$$
\mu_{\eta}=\bar{g}\langle x\rangle_{\eta}, \quad C_{\eta}(\tau)=g^{2}\langle x x\rangle_{\eta}(\tau)
$$

The averages $\langle x\rangle_{\eta} \equiv v$ and $\langle x x\rangle_{\eta}(\tau)-v^{2} \equiv C_{x}(\tau)$ denote the mean (firing rate) and correlation function of the spike train produced by a neuron driven by the effective stochastic input $\eta(t)$. Since the input thereby appears on both the left-hand and the right-hand sides, this poses a self-consistency problem.

To recapitulate, DMFT approximates the input of a single neuron by an effective Gaussian process with self-consistent statistics [Fig. 1(b)]. Thus the description, albeit stochastic, is still on the level of individual neurons. These individual neurons driven by Gaussian processes form an ensemble with the same statistics across neurons as the original network. In particular, this means that population-averaged quantities, e.g., the autocorrelation function, but also distributions across the neurons, e.g., the distribution of the firing rate, can be computed from the DMFT.

\section{Static contribution}

The networks we consider are heterogeneous even within a population-each neuron potentially has a different number of presynaptic partners and thus also a different firing rate [50]. On a first glance, DMFT neglects this heterogeneity. However, Eqs. (7) in fact account for such static variabilities: on the right-hand side the second moment of the spike train appears instead of the correlation function. Rewriting $\langle x x\rangle_{\eta}(\tau)=C_{x}(\tau)+v^{2}$ reveals a first static component $g^{2} v^{2}$ of the variability of the effective input due to the firing rate of individual neurons. Moreover, $C_{x}(\tau \rightarrow \infty) \equiv \sigma_{v}^{2}$ potentially saturates on a plateau which accounts for the variability of the firing rate across neurons [Fig. 1(c)]. To make this explicit, we sometimes rewrite

$$
\eta(t)=\zeta+\xi(t)
$$

where $\zeta$ is a Gaussian random variable with $\mu_{\zeta}=\bar{g} v, \sigma_{\zeta}^{2}=$ $g^{2}\left(v^{2}+\sigma_{v}^{2}\right)$ and $\xi(t)$ a zero-mean Gaussian process with $C_{\xi}(\tau)=g^{2}\left(C_{x}(\tau)-\sigma_{v}^{2}\right)$.

\section{B. Multiple populations}

Using the expressions Eqs. (7) for a single population, we can straightforwardly generalize the theory to multiple populations. Due to the independence of the effective inputs in DMFT, both mean and correlation function are a simple sum over the contributions from all populations [18,51]:

$$
\begin{gathered}
\mu_{\eta}^{\alpha}=\sum_{\beta} \bar{g}^{\alpha \beta} v^{\beta}, \\
C_{\eta}^{\alpha}(\tau)=\sum_{\beta}\left(g^{\alpha \beta}\right)^{2}\left(C_{x}^{\beta}(\tau)+\left(v^{\beta}\right)^{2}\right),
\end{gathered}
$$

with the corresponding generalizations of Eqs. (6), $\bar{g}^{\alpha \beta}=$ $K^{\alpha \beta} \mu_{J}^{\alpha \beta}$ and $\left(g^{\alpha \beta}\right)^{2}=K^{\alpha \beta}\left(\left(\sigma_{J}^{\alpha \beta}\right)^{2}+\left(1-p^{\alpha \beta}\right)\left(\mu_{J}^{\alpha \beta}\right)^{2}\right)$. This leads to one stochastic equation per population [Fig. 1(b)]. As before, we can split the input into static and dynamic contributions, $\eta^{\alpha}(t)=\zeta^{\alpha}+\xi^{\alpha}(t)$.

\section{External input}

We take the sum $\sum_{\beta}$ to include external populations, e.g., excitatory neurons that drive the network dynamics with homogeneous Poissonian spike trains of rate $v^{\text {ext }}$. In Eqs. (9) and (10), such an external Poisson input leads to a term $J^{\alpha, \text { ext }} v^{\text {ext }}$ and $\left(J^{\alpha, \text { ext }}\right)^{2} \nu^{\text {ext }} \delta(\tau)$, respectively. If the network is driven by a constant external input, only Eq. (9) obtains an additional contribution $\mu_{\mathrm{ext}}^{\alpha}$. An external zero-mean, stationary Gaussian process leads to an additional term $C_{\text {ext }}(\tau)$ in Eq. (10). 


\section{Output statistics}

Approximating the input is only the first step. In a second step, the self-consistency problem has to be solved. To this end, the output statistics of a neuron driven by a nonMarkovian Gaussian process have to be calculated. In other words, we need a solution for the colored noise problem. The full non-Markovian problem has to be considered because a Markovian approximation neglects the quantity of interest: the temporal correlations. For sufficiently simple rate neurons, the problem is analytically solvable $[15,52]$; the case of two spiking neuron models is discussed in the following sections. For the remainder of this section, let us assume that we are able to solve the colored noise problem to obtain a self-consistent solution of Eqs. (9) and (10).

\section{Timescale}

Given a self-consistent solution, we can calculate the intrinsic timescale from the spike-train autocorrelation function $C_{x}^{\alpha}(\tau)$. Since $C_{x}^{\alpha}(\tau)$ always contains a delta peak [40], we consider only the smooth part of the autocorrelation function $\hat{C}_{x}^{\alpha}(\tau) \equiv C_{x}^{\alpha}(\tau)-v^{\alpha} \delta(\tau)$. To characterize the timescale, we use the definition of Ref. [40] [Fig. 1(c)]:

$$
\tau_{c}^{\alpha}=\int_{0}^{\infty}\left|\frac{\hat{C}_{x}^{\alpha}(\tau)-\hat{C}_{x}^{\alpha}(\infty)}{\hat{C}_{x}^{\alpha}(0)-\hat{C}_{x}^{\alpha}(\infty)}\right| d \tau .
$$

Note that the definition of the autocorrelation time is not unequivocal. Other possible definitions include $\tau_{c}^{\alpha}=\int_{-\infty}^{\infty}\left|\frac{\hat{C}_{x}^{\alpha}(\tau)-\hat{C}_{x}^{\alpha}(\infty)}{\hat{C}_{x}^{\alpha}(0)-\hat{C}_{x}^{\alpha}(\infty)}\right|^{2} d \tau$ [37] and $\tau_{c}^{\alpha}=\frac{\int_{0}^{\infty} \tau\left|\hat{C}_{x}^{\alpha}(\tau)-\hat{C}_{x}^{\alpha}(\infty)\right| d \tau}{\int_{0}^{\infty}\left|\hat{C}_{x}^{\alpha}(\tau)-\hat{C}_{x}^{\alpha}(\infty)\right| d \tau}$ [23]. We observed drastic differences between these definitions for empirical correlation functions directly obtained from the simulations. These differences are in part an artifact from the absolute value: the variance of the empirical estimate grows with $\tau$ [53]; due to the absolute value these fluctuations add up. The three functional forms carry with them different fluctuations, e.g., the squared fluctuations $\left|\frac{\hat{C}_{x}^{\alpha}(\tau)-\hat{C}_{x}^{\alpha}(\infty)}{\hat{C}_{x}^{\alpha}(0)-\hat{C}_{x}^{\alpha}(\infty)}\right|^{2}$ are typically much smaller than $\left|\frac{\hat{C}_{x}^{\alpha}(\tau)-\hat{C}_{x}^{\alpha}(\infty)}{\hat{C}_{x}^{\alpha}(0)-\hat{C}_{x}^{\alpha}(\infty)}\right|<1$, and hence lead to different estimates. For theoretically predicted autocorrelations, the difference is less drastic and we choose Eq. (11) because it is the most simple definition. Due to this difficulty, we always use the theoretical prediction of the autocorrelation function to determine the timescale-after checking that it matches the empirical autocorrelation function well apart from fluctuations.

In addition to $\tau_{c}^{\alpha}$, we will also consider the asymptotic decay constant [Fig. 1(c)]

$$
\hat{C}_{x}^{\alpha}(\tau)-\hat{C}_{x}^{\alpha}(\infty) \sim \exp \left(-\tau / \tau_{\infty}^{\alpha}\right),
$$

because in special cases $\tau_{\infty}^{\alpha}$ directly follows from our theory. For a simple exponential autocorrelation function, the timescales in Eqs. (11) and (12) coincide. We work from the assumption that Eq. (12) is a good approximation to Eq. (11) and verify this assumption post hoc.

In the literature, there are even more definitions of intrinsic timescales than the ones mentioned above. For example, [2] assume an exponential correlation function and an offset, similar to Eq. (12) but for all time lags and not just asymptotically. In contrast, Ref. [54] determine the timescale by fitting a
Lorentzian to the power spectrum after removing oscillatory components. Yet another approach, determining the half width at half maximum of the autocorrelation function, is advocated for in Ref. [55]. To avoid these ambiguities, we use the established definitions, Eqs. (11) and (12), from the stochastic processes literature.

Recently, two new approaches have been proposed to estimate the timescale directly from spiking data $[38,56]$. While both overcome important challenges, biases in the estimated timescale related to and independent of subsampling, respectively, we do not use them here because they rely on models which implicitly assume (a mixture of) exponential correlation functions: Ref. [56] assumes an autoregressive model and Ref. [38] a mixture of Ornstein-Uhlenbeck processes.

\section{Spike train power spectrum}

Instead of the autocorrelation function, we sometimes consider the spike train power spectrum [Fig. 1(d)]

$$
S_{x}^{\alpha}(f)=\int_{-\infty}^{\infty} e^{2 \pi i f \tau} C_{x}^{\alpha}(\tau) d \tau
$$

Due to the delta peak in the autocorrelation function, the power spectrum always saturates at the firing rate, $S_{x}^{\alpha}(f) \stackrel{f \rightarrow \infty}{\rightarrow}$ $v^{\alpha}$. For a renewal process, the zero-frequency limit is $S_{x}^{\alpha}(f) \stackrel{f \rightarrow 0}{\rightarrow} v^{\alpha} \mathrm{CV}_{\alpha}^{2}[24]$, which directly reveals the coefficient of variation of the interspike-interval (ISI) distribution $\mathrm{CV}_{\alpha}$.

\section{Comparison with simulations}

In our theory, we consider disorder-averaged quantities and stationary processes. To compare the theory with a single simulation, we assume self-averaging in the sense that the activity distribution across neurons is approximately the same for each network realization. Since neurons with different indegrees have different disorder- and time-averaged inputs, in practice this means that we assume that neurons with comparable indegree have comparable activity statistics in each network realization.

The disorder averages preserve the static variability across neurons, as we consider the same connectivity statistics, and in particular the same indegree distribution, across realizations. Self-averaging works well when each neuron (or at least a sufficiently large proportion of neurons) receives input from a representative sample of the rest of the network.

Under stationarity, distributions across neurons of instantaneous rates at any given time point (but not of instantaneous rates across time points - which we do not consider here) equal distributions of time-averaged rates across neurons. To obtain the rate distributions from the simulations, we use timeaveraged rates to reduce the variance of the corresponding estimates. Similarly, we use time averages to compute the single-neuron autocorrelation functions and power spectra.

We focus on the second-order statistics. Since first-order statistics, i.e., the firing rate, scale the power spectra and correlation function [24], we plot $S_{x}(f) / v$ and $C_{x}(f) / \nu^{2}$ to eliminate this trivial dependency. Note that a multiplicative factor does not influence the intrinsic timescale, Eq. (11). 


\section{GENERALIZED LINEAR MODEL NEURONS}

First, we consider generalized linear model (GLM) neurons [24,57]. GLM neurons are stochastic model neurons that spike according to an inhomogeneous Poisson process at a rate determined by the synaptic input. Due to their simplicity, GLM neurons are frequently fitted to experimental data [24]; here we consider them because they are analytically tractable.

\section{A. Neuron dynamics}

Each neuron generates a spike train according to an inhomogeneous Poisson process with intensity (rate)

$$
\lambda_{i}^{\alpha}(t)=c_{1}^{\alpha} \phi\left[c_{2}^{\alpha}\left(V_{i}^{\alpha}(t)-\theta^{\alpha}\right)\right],
$$

where $\theta^{\alpha}$ denotes the (soft) threshold, $\phi(V)$ is a smooth, nonnegative, monotonically increasing function, and $c_{1}^{\alpha}>0$, $c_{2}^{\alpha}>0$ are free parameters. The voltage is given by a linear filtering of the input

$$
V_{i}^{\alpha}(t)=\int_{-\infty}^{\infty} \kappa^{\alpha}(t-s) \eta_{i}^{\alpha}\left(s-d^{\alpha \beta}\right) d s,
$$

where $d^{\alpha \beta}$ allows for a transmission delay. For all simulations, we choose a filter with a single exponential with time constant $\tau_{\mathrm{m}}^{\alpha}$, which corresponds to post-synaptic currents in the form of delta spikes:

$$
\kappa^{\alpha}(t)=\Theta(t) e^{-t / \tau_{\mathrm{m}}^{\alpha}} .
$$

Here, $\Theta(t)$ denotes the Heaviside function ensuring causality of the filter. We rescale the synaptic weights $J_{i j}^{\alpha \beta}$ and the threshold $\theta^{\alpha}$ using $c_{2}^{\alpha}$ such that $c_{2}^{\alpha}=1$ throughout the rest of this section.

\section{Colored noise problem}

The effective stochastic input $\eta^{\alpha}(t)$ leads to stochastic voltage dynamics. Because the voltage is given by a convolution, the voltage becomes a Gaussian process with

$$
\mu_{V}^{\alpha}=\bar{\kappa}^{\alpha} \mu_{\eta}^{\alpha}, \quad C_{V}^{\alpha}(\tau)=\int_{-\infty}^{\infty} \tilde{\kappa}^{\alpha}(\tau-s) C_{\eta}^{\alpha}(s) d s,
$$

where the filter determines $\bar{\kappa}^{\alpha}=\int_{-\infty}^{\infty} \kappa^{\alpha}(t) d t$ and $\tilde{\kappa}^{\alpha}(t)=\int_{-\infty}^{\infty} \kappa^{\alpha}(s) \kappa^{\alpha}(s-t) d s$. For the single-exponential filter that we used in simulations, we have $\bar{\kappa}^{\alpha}=\tau_{\mathrm{m}}^{\alpha}$ and $\tilde{\kappa}^{\alpha}(t)=\frac{\tau_{\mathrm{m}}^{\alpha}}{2} e^{-|t| / \tau_{\mathrm{m}}^{\alpha}}$. Note that the transmission delay cancels in the stationary case considered here.

All cumulants of the resulting spike trains $x(t)$ can be obtained from their characteristic functional [40] [see Appendix A, Eq. (A8)]:

$$
\Phi_{x}[u(t)]=\exp \left(\int_{0}^{T}\left(e^{i u(t)}-1\right) \lambda(t) d t\right) .
$$

From here, we temporarily drop the population index for the sake of clarity. Averaging over realizations of the rates yields

$$
\begin{aligned}
\left\langle\Phi_{x}[u(t)]\right\rangle_{\lambda} \approx & e^{\int_{0}^{T}\left(e^{i u(t)}-1\right) \mu_{\lambda}(t) d t} \\
& \times e^{\frac{1}{2} \int_{0}^{T} \int_{0}^{T}\left(e^{i u(t)}-1\right) C_{\lambda}\left(t, t^{\prime}\right)\left(e^{i u\left(t^{\prime}\right)}-1\right) d t d t^{\prime}},
\end{aligned}
$$

where $\mu_{\lambda}(t)$ denotes the mean of $\lambda(t), C_{\lambda}\left(t, t^{\prime}\right)$ its correlation function, and we neglect terms of $O\left(u^{3}\right)$ since we are only interested in the first and second cumulants. Expanding also $e^{i u(t)}-1$ to second order in $u(t)$, we can simply read off the stationary cumulants

$$
v=\mu_{\lambda}, \quad C_{x}(\tau)=\mu_{\lambda} \delta(\tau)+C_{\lambda}(\tau),
$$

in agreement with the result of Ref. [58].

We are left with the task of calculating the first two cumulants of $\lambda(t)$ from $\mu_{V}$ and $C_{V}(\tau)$, depending on the choice of the nonlinearity $\phi(V)$.

\section{Exponential nonlinearity}

First, we consider the commonly employed exponential nonlinearity [24]

$$
\phi(V)=\exp (V) .
$$

Both cumulants are straightforward to obtain from the characteristic functional of the voltage. We have [see Appendix A, Eqs. (A4) and (A5)]

$$
\begin{aligned}
\left\langle\phi\left(V\left(t_{1}\right)\right)\right\rangle_{V} & =\left\langle e^{\int_{0}^{T} V(t) \delta\left(t-t_{1}\right) d t}\right\rangle_{V} \\
& =e^{\mu_{V}+\frac{1}{2} C_{V}(0)}, \\
\left\langle\phi\left(V\left(t_{1}\right)\right) \phi\left(V\left(t_{2}\right)\right)\right\rangle_{V} & =\left\langle e^{\int_{0}^{T} V(t)\left[\delta\left(t-t_{1}\right)+\delta\left(t-t_{2}\right)\right] d t}\right\rangle_{V} \\
& =e^{2 \mu_{V}+C_{V}(0)+C_{V}\left(t_{2}-t_{1}\right)},
\end{aligned}
$$

where we used the stationarity of $V$. Including the prefactor and the threshold from Eq. (14), we get

$$
\begin{gathered}
\mu_{\lambda}=c_{1} \exp \left(\mu_{V}-\theta+\frac{1}{2} C_{V}(0)\right), \\
C_{\lambda}(\tau)=\mu_{\lambda}^{2} \exp \left(C_{V}(\tau)\right)-\mu_{\lambda}^{2} .
\end{gathered}
$$

From Eq. (21), it follows that $C_{\lambda}(\tau)$ has a static part as long as $C_{V}(\infty)>0$. Since $C_{\eta}(\tau)$ contains a static part [see Eqs. (8) and (10)], $C_{V}(\tau)$ and hence $C_{\lambda}(\tau)$ and $C_{x}(\tau)$ indeed also contain a static contribution and saturate on a plateau.

Rate distribution. The rate distribution across neurons is lognormal because the (static) input distribution is Gaussian and the f-I curve is a simple exponential [50]. The theory yields the mean $v=c_{1} \exp \left(\mu_{V}-\theta+\frac{1}{2} C_{V}(0)\right)$ and variance $\sigma_{v}^{2}=C_{x}(\infty)=v^{2}\left(e^{C_{V}(\infty)}-1\right)$ of the firing rate. We note that we can obtain the same result from a constant input with mean $\tilde{\mu}_{V}=\mu_{V}-\theta+\frac{1}{2} C_{V}(0)-\frac{1}{2} C_{V}(\infty)$ and variance across neurons $\tilde{\sigma}_{V}^{2}=C_{V}(\infty)$. Parameterized in terms of $\tilde{\mu}_{V}$ and $\tilde{\sigma}_{V}$, the firing rate distribution is thus

$$
p(v)=v^{-1} \mathcal{N}\left(\ln \left(v / c_{1}\right) \mid \tilde{\mu}_{V}, \tilde{\sigma}_{V}^{2}\right)
$$

with the normal distribution $\mathcal{N}\left(x \mid \mu, \sigma^{2}\right)$.

\section{Error function nonlinearity}

A drawback of the exponential function, Eq. (19), is that it allows for infinite rates. Thus we also consider the bounded nonlinearity

$$
\phi(V)=\frac{1}{2}(1+\operatorname{erf}(V / \sqrt{2})) .
$$

The integrals to determine the cumulants can be solved using the table [59] (details in Appendix B 1); the result 
is

$$
\begin{gathered}
\mu_{\lambda}=\frac{c_{1}}{2}(1+\operatorname{erf}(h / \sqrt{2})), \\
C_{\lambda}(\tau)=c_{1} \mu_{\lambda}-2 c_{1}^{2} T(h, a(\tau))-\mu_{\lambda}^{2},
\end{gathered}
$$

where we again suppressed the population index, abbreviated $h=\frac{\mu_{V}-\theta}{\sqrt{1+C_{V}(0)}}$ and $a(\tau)=\left(\frac{1+C_{V}(0)-C_{V}(\tau)}{1+C_{V}(0)+C_{V}(\tau)}\right)^{1 / 2}$, and used Owen's $\mathrm{T}$ function $T(h, a)=\frac{1}{2 \pi} \int_{0}^{a} d x \frac{e^{-\frac{1}{2} h^{2}\left(1+x^{2}\right)}}{1+x^{2}}$.

Rate distribution. Equivalent to the situation for the exponential nonlinearity, the input distribution across neurons is Gaussian. Again, we consider the equivalent static problem which, in this case, leads to $\tilde{\mu}_{V}=\frac{\mu_{V}-\theta}{\sqrt{1+C_{V}(0)-C_{V}(\infty)}}$ and $\tilde{\sigma}_{V}^{2}=\frac{C_{V}(\infty)}{1+C_{V}(0)-C_{V}(\infty)}$. Parameterized in terms of $\tilde{\mu}_{V}$ and $\tilde{\sigma}_{V}$, the firing rate distribution is

$$
p(v)=\frac{\mathcal{N}\left(\operatorname{probit}\left(\nu / c_{1}\right) \mid \tilde{\mu}_{V}, \tilde{\sigma}_{V}^{2}\right)}{c_{1} \mathcal{N}\left(\operatorname{probit}\left(\nu / c_{1}\right) \mid 0,1\right)},
$$

where $\operatorname{probit}(x)$ denotes the inverse of the standard normal cumulative distribution, i.e., probit $(\phi(V))=V$, and we used $\phi^{\prime}(V)=\mathcal{N}(V \mid 0,1)$.

\section{Numerical solution of the self-consistency problem}

We solve the self-consistency problem using a fixed-point iteration [32,35]. To initiate the algorithm, we set $v^{\alpha}=\frac{1}{2} c_{1}^{\alpha}$ and $C_{\lambda}^{\alpha}(t)=0$. Next, we determine the input statistics according to Eqs. (9) and (10); then we determine the voltage statistics according to (17). From the voltage statistics, we can obtain the statistics of the rate via Eqs. (20) and (21) [or Eqs. (24) and (25)]. Denoting the rate thus calculated as $\hat{\mu}_{\lambda, n+1}^{\alpha}$, we then update the rate statistics using incremental steps, $\mu_{\lambda, n+1}^{\alpha}=\mu_{\lambda, n}^{\alpha}+\varepsilon\left(\hat{\mu}_{\lambda, n+1}^{\alpha}-\mu_{\lambda, n}^{\alpha}\right)$ for the mean rate, and similarly for all entries of $C_{\lambda}^{\alpha}(t)$. The new firing rate statistics lead via (18) to new spike train statistics. Here, the small update step $\varepsilon<1$ is crucial because otherwise the fixed-point iteration is numerically unstable. Now we iterate and generate new voltage statistics. With the incremental update and the initialization $v^{\alpha}=\frac{1}{2} c_{1}^{\alpha}$, the algorithm quickly converged to the fixed point corresponding to the simulation in the examples we considered. Due to the analytical solutions, the only bottleneck for the numerics is the convolution in Eq. (17), which can be solved efficiently using the fast Fourier transform [60]. Thus, even the parameter scans with 5000 points described in the following run on a laptop in less than two minutes.

\section{B. Balanced random network}

As a first application of the theory, we consider a balanced random network of excitatory and inhibitory GLM neurons. The network contains two populations [Fig. 2(a)], $\alpha \in\{E, I\}$, and it is driven by an excitatory external input which we incorporate into an effective threshold $\theta_{\text {eff }}=\theta-\mu_{\text {ext }}$. Here, we use a constant external input rather than a Poisson drive because we are particularly interested in finding long timescales, which might be hindered by the lack of temporal correlation of Poisson spike trains. However, the theory can straightforwardly be applied to Poisson input. Although four times more excitatory cells are present in the network, we typically place it in an inhibition-dominated regime by increasing the (a)

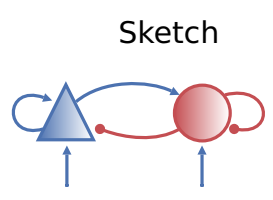

(c)
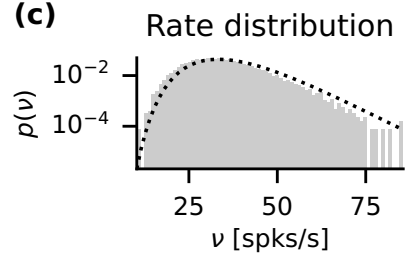

(b)

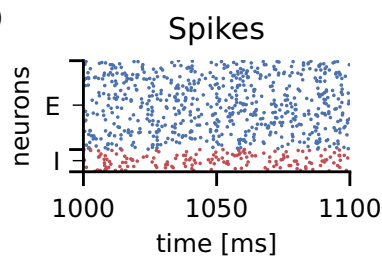

(d)

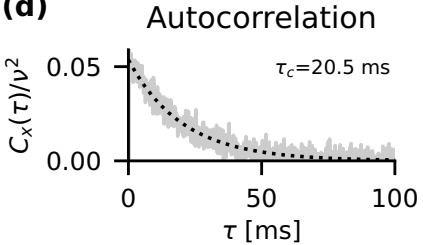

FIG. 2. Balanced random network of GLM neurons with exponential nonlinearity. (a) Sketch of the network with populations of excitatory (blue) and inhibitory (red) neurons. (b) Raster plot of $2 \%$ of the excitatory (blue) and inhibitory (red) neurons. (c) Firing rate distribution across all neurons from simulation (gray) and theory (black) using Eq. (22). (d) Population-averaged single-unit autocorrelation function from simulation (gray) and self-consistent theory (black) using Eqs. (20) and (21). Here, we subtracted the static contribution $C_{x}(\infty)$. Parameters: $N_{E}=10000, N_{I}=2500$, $J_{E}=0.25 \mathrm{mV},\left|J_{I} / J_{E}\right|=4.5, p=0.1, \tau_{\mathrm{m}}=20 \mathrm{~ms}, \theta_{\text {eff }}=0 \mathrm{mV}$, $c_{1}=50 \mathrm{~s}^{-1}, c_{2}=0.02 \mathrm{mV}^{-1}$, and $d=1.5 \mathrm{~ms}$.

synaptic weights of the inhibitory neurons. As well known [26], this settles the network in the balanced state leading to asynchronous irregular activity of the neurons [see, e.g., Fig. 2(b)].

In line with Brunel's model A [26], we choose identical values for the single-neuron parameters. Since we also choose the same connection probability of $10 \%$ for all pairs of populations, both populations receive statistically identical input in the DMFT approximation. Due to identical singleneuron parameters and input statistics, the statistics of the activity is the same for excitatory and inhibitory neurons [see, e.g., Fig. 2(b)]; therefore, we do not distinguish between the populations for the statistics in our plots. In contrast to the network examined by Brunel, we consider the somewhat more involved case of a fixed connection probability between a pair of neurons instead of a fixed number of incoming synapses per neuron (indegree). The fixed connection probability leads to a (binomially) distributed indegree across neurons, such that a strong variability across neurons is present in the network [see, e.g., Fig. 2(c)]. This variability is already present on the level of mean firing rates, i.e., there is static variability in the network.

All simulations were performed using the NEST simulator version 2.20.1 [61]. In all GLM network simulations, we simulated $1 \mathrm{~min}$ of biological time with a time step of $0.1 \mathrm{~ms}$ and discarded an initial transient of $1 \mathrm{~s}$. For the GLM neurons, we used the "pp_psc_delta" neuron model. To allow for the error function nonlinearity, we modified the "pp_psc_delta" model accordingly.

\section{Exponential nonlinearity: absence of long timescales}

First, we consider networks with an exponential nonlinearity (Fig. 2). The fixed-point iteration yields a rate distribution 


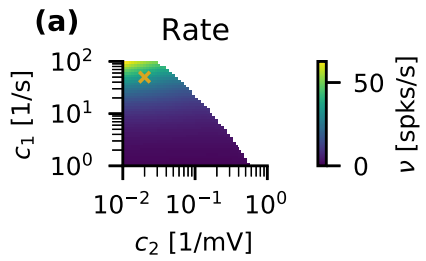

(b) Timescale

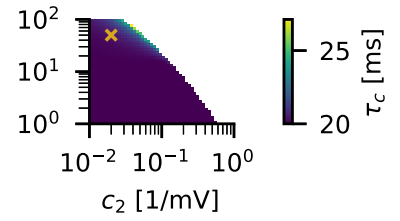

(c)
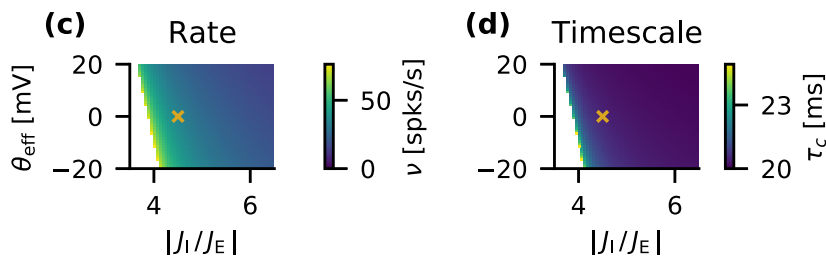

FIG. 3. Parameter scan for a balanced random network of GLM neurons with exponential nonlinearity. [(a) and (b)] Firing rate and intrinsic timescale for varying neuron parameters $c_{1}$ and $c_{2}$. Parameters used in (c) and (d) and Fig. 2 indicated by orange crosses. [(c) and (d)] Firing rate and intrinsic timescale for varying effective threshold $\theta_{\text {eff }}$ and relative inhibitory strength $\left|J_{I} / J_{E}\right|$. Parameters used in (a) and (b) and Fig. 2 indicated by orange crosses. Further parameters as in Fig. 2.

and an autocorrelation function that closely match the simulation [Figs. 2(c) and 2(d)]. The theory for the rate distribution [Fig. 2(c)] is slightly biased towards higher rates; a possible cause for this is a finite size effect because the mean inhibitory indegree $K_{I}=p N_{I}=250$ is relatively small. Nonetheless, the theory predicts the autocorrelation function very well [Fig. 2(d)] and yields a timescale $\tau_{c} \approx \tau_{\mathrm{m}}=20 \mathrm{~ms}$.

For the parameters in Fig. 2, the intrinsic timescale is close to the membrane time constant. This raises the question whether longer timescales can be achieved in a network of GLM neurons. To answer this question, we employ our theory and perform parameter scans. First, we vary the single-neuron parameters $c_{1}$ and $c_{2}$ [Figs. 3(a) and 3(b)]. The rate increases monotonically with $c_{1}$ while $c_{2}$ has as smaller effect up to a certain threshold [Fig. 3(a)]. Beyond this threshold, the rate diverges rapidly to infinity in the threshold iteration [white area in Fig. 3(a)]. The timescale is close to the membrane time constant throughout the nondivergent regime and only increases slightly towards the threshold where the rate diverges [Fig. 3(b)]. Next, we vary the strength of the external input by adjusting the effective threshold $\theta_{\text {eff }}$ and the inhibition dominance by varying $\left|J_{I} / J_{E}\right|$ for constant $J_{E}$. We find a clear threshold of $\left|J_{I} / J_{E}\right|$ beyond which the rate diverges [Fig. 3(c)]. Again, this threshold corresponds to the regime where the timescale slowly starts to grow above the membrane time constant.

Put together, these observations suggest that the rate divergence prevents recurrent dynamics with long timescales in balanced random networks of GLM neurons with exponential nonlinearity.

\section{Error function nonlinearity: existence of long timescales}

In the previous section, the rate divergence prevented long timescales. To avoid the divergence, we consider the bounded transfer function Eq. (23) and use our theory for parameter scans (Fig. 4). The effect of the single-neuron

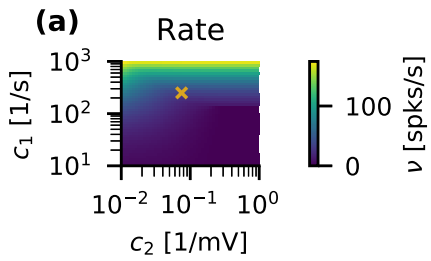

(b) Timescale
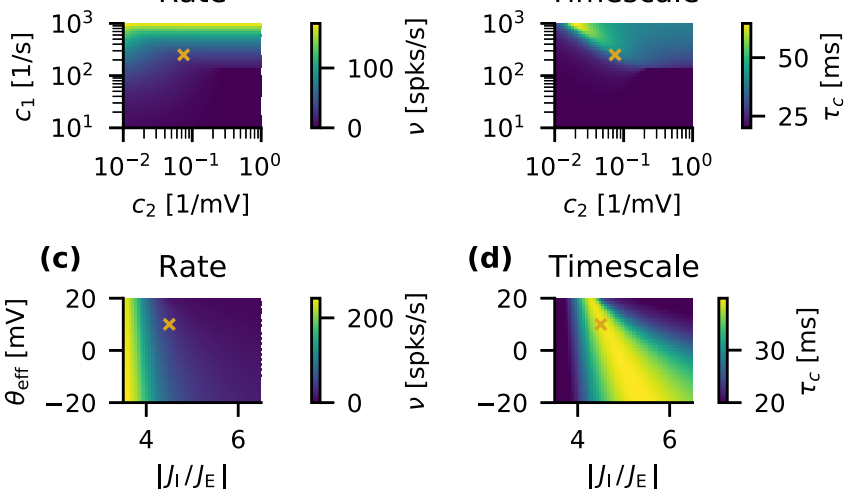

(d) Timescale

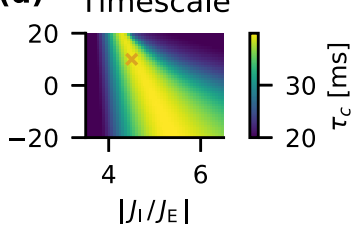

FIG. 4. Parameter scan for a balanced random network of GLM neurons with error function nonlinearity. [(a) and (b)] Firing rate and intrinsic timescale for varying neuron parameters $c_{1}$ and $c_{2}$. Parameters used in (c) and (d) and Fig. 5 indicated by orange crosses. [(c) and (d)] Firing rate and intrinsic timescale for varying effective threshold $\theta_{\text {eff }}$ and relative inhibitory strength $\left|J_{I} / J_{E}\right|$. Parameters used in (a) and (b) and Fig. 5 indicated by orange crosses. Further parameters as in Fig. 2.

parameters $c_{1}$ and $c_{2}$ is similar to the unbounded case but the rate divergence is absent [Fig. 4(a)]. This allows for a parameter regime with longer timescales up to approximately $3 \tau_{\mathrm{m}}$ [Fig. 4(b)]. Similarly, varying $\theta_{\text {eff }}$ and $\left|J_{I} / J_{E}\right|$ uncovers a regime with a rate close to the maximum $c_{1}$ when the network is not inhibition-dominated [Fig. 4(c)]. Outside the inhibition-dominated regime, we expect that our theory does not yield quantitatively accurate predictions. The effect on the timescale is more subtle: within the inhibition-dominated regime, for any given $\left|J_{I} / J_{E}\right|$ the timescale displays a maximum whose location depends on the external input [Fig. 4(d)].

What kind of dynamics is displayed by the network at such a local maximum of the timescale? The corresponding spike trains show a strong variability of firing rate across neurons and temporally correlated spikes [Fig. 5(a)]. The rate distribution reveals that all rates between the minimum zero and the maximum $c_{1}$ are present, in excellent agreement with the theoretical prediction [Fig. 5(b)]. In the example considered, the empirical estimate of the network-averaged single-unit autocorrelation displays an intrinsic timescale of approximately $2 \tau_{\mathrm{m}}$; again, the empirical estimate and the theoretical prediction agree closely [Fig. 5(c)]. From the spike train power spectrum, a high $\mathrm{CV}>2$ is apparent [Fig. 5(d)]. All of these characteristics agree with the "heterogeneous asynchronous state" uncovered in [62].

\section{Error function nonlinearity: mechanism of timescale}

To uncover the mechanisms that shape the timescale, in particular the local maximum in Fig. 4(d), we develop a theory for the asymptotic timescale $\tau_{\infty}$, Eq. (12). To this end, we use that $\tilde{\kappa}(t)=\frac{\tau_{\mathrm{m}}}{2} e^{-|t| / \tau_{\mathrm{m}}}$ is the fundamental solution to the differential operator $1-\tau_{\mathrm{m}}^{2} \frac{d^{2}}{d t^{2}}$, i.e., $\left(1-\tau_{\mathrm{m}}^{2} \frac{d^{2}}{d t^{2}}\right) \tilde{\kappa}(t)=\tau_{\mathrm{m}}^{2} \delta(t)$. Thus we can rewrite Eq. (17) into a differential equation:

$$
\tau_{\mathrm{m}}^{2} \ddot{C}_{V}=C_{V}-\tau_{\mathrm{m}}^{2} C_{\eta}
$$


(a)

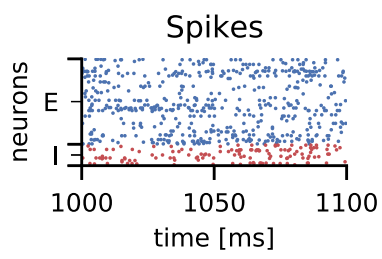

(c)

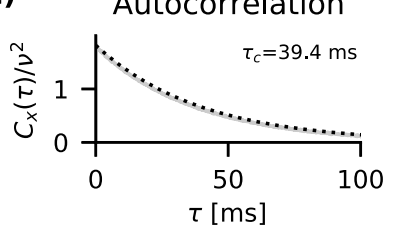

(b) Rate distribution

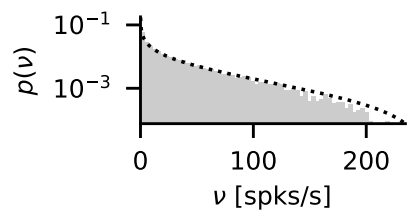

(d)

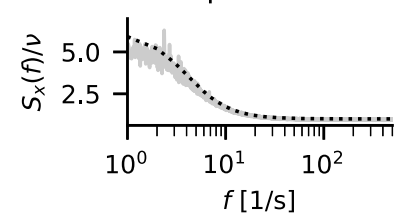

FIG. 5. Balanced random network of GLM neurons with error function nonlinearity. (a) Raster plot of $2 \%$ of the excitatory (blue) and inhibitory (red) neurons. (b) Firing rate distributions across all neurons from simulation (gray) and theory (black) using Eq. (26). [(c) and (d)] Population-averaged single-unit autocorrelation function and power spectrum from simulation (gray) and self-consistent theory (black) using Eqs. (24) and (25). As in Fig. 2, we subtracted the static contribution $C_{x}(\infty)$. Parameters: $c_{1}=250 \mathrm{~s}^{-1}$, $c_{2}=0.075 \mathrm{mV}^{-1}$, and further parameters as in Fig. 2 .

where the dependence of $C_{\eta}$ on $C_{V}$ is determined by Eqs. (10), (18), and (25). Next, we rescale time such that $\tau_{\mathrm{m}}=1$ and linearize this differential equation for small $\Delta_{V}(\tau) \equiv C_{V}(\tau)-$ $C_{V}(\infty)$ to obtain

$$
\ddot{\Delta}_{V}=\left(1-\frac{d C_{\eta}(\infty)}{d C_{V}(\infty)}\right) \Delta_{V}+O\left(\Delta_{V}^{2}\right) .
$$

This allows for an exponential solution with time constant

$$
\tau_{\infty}=\frac{1}{\sqrt{1-g^{2} \frac{d C_{\lambda}(\infty)}{d C_{V}(\infty)}}}
$$

where we used Eqs. (10) and (18) to derive $\frac{d C_{\eta}(\infty)}{d C_{V}(\infty)}=g^{2} \frac{d C_{\lambda}(\infty)}{d C_{V}(\infty)}$. We see that there are two factors that determine the timescale: the cumulant of the connectivity $g^{2}$ and the gain of the rate autocorrelation $\frac{d C_{\lambda}(\infty)}{d C_{V}(\infty)}$. For the latter, we obtain from Eq. (25)

$$
\frac{d C_{\lambda}(\infty)}{d C_{V}(\infty)}=\frac{c_{1}^{2}}{2 \pi} \frac{\exp \left(-\frac{\left(\mu_{V}-\theta_{\text {eff }}\right)^{2}}{1+C_{V}(0)+C_{V}(\infty)}\right)}{\sqrt{\left(1+C_{V}(0)\right)^{2}-C_{V}(\infty)^{2}}} .
$$

Thus, given a self-consistent autocorrelation $C_{x}$ and the corresponding voltage statistics from Eq. (17), the asymptotic timescale Eq. (27) can be directly evaluated.

We vary $\theta_{\text {eff }}$ and $\left|J_{I} / J_{E}\right|$ in Figs. 6(a)-6(c). First, we plot $\frac{d C_{\lambda}(\infty)}{d C_{V}(\infty)}$ alone, which we refer to as the gain [Fig. 6(a)]. Due to the interplay between the exponential suppression $\exp \left(-\frac{\left(\mu_{V}-\theta_{\text {eff }}\right)^{2}}{1+C_{V}(0)+C_{V}(\infty)}\right)$ and the square root factor $1 / \sqrt{\left(1+C_{V}(0)\right)^{2}-C_{V}(\infty)^{2}}<1$ in Eq. (28), the gain already exhibits a maximum. The existence of the maximum is mainly determined by the exponential suppression with growing $\left|\mu_{V}-\theta_{\text {eff }}\right|$ in Eq. (28): in both the excitation- and the inhibition-dominated regimes, $\mu_{V}$ is far from the effective threshold $\theta_{\text {eff }}$. The precise location of the maximum is
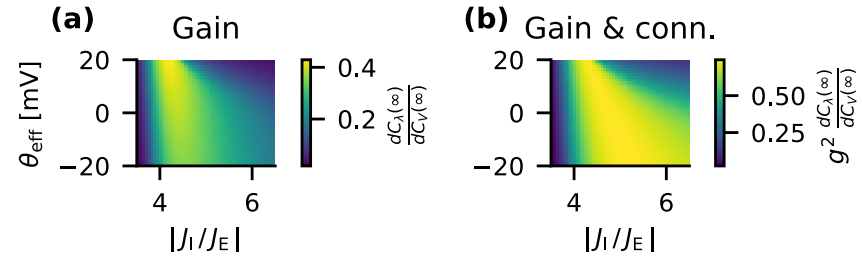

(c) Timescale

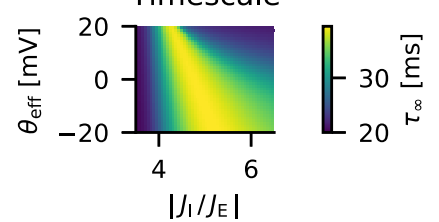

(d) Timescale

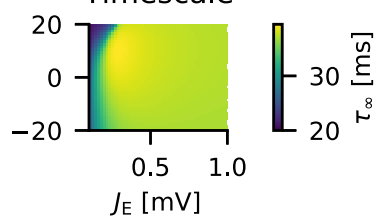

FIG. 6. Mechanisms that shape the asymptotic timescale. (a) Asymptotic gain $\frac{d C_{\lambda}(\infty)}{d C_{V}(\infty)}$ of the rate autocorrelation w.r.t. changes in the voltage autocorrelation, Eq. (28). (b) Asymptotic gain multiplied by the second cumulant of the connectivity, $g^{2}$. (c) Asymptotic timescale according to Eq. (27), $\tau_{\infty}=\left(1-g^{2} \frac{d C_{\lambda}(\infty)}{d C_{V}(\infty)}\right)^{-1 / 2}$, for varying effective threshold $\theta_{\text {eff }}$ and relative inhibitory strength $\left|J_{I} / J_{E}\right|$. (d) Same as $\mathbf{c}$ for varying excitatory synaptic strength $J_{E}$ with constant $\left|J_{I} / J_{E}\right|$. Further parameters as in Fig. 5.

not necessarily at $\mu_{V}=\theta_{\text {eff }}$ as it is also determined by the square root factor. The latter decays reciprocally to $C_{V}(0)$ and $C_{V}(\infty)$. Both $C_{V}(0)$ and $C_{V}(\infty)$ decay for growing effective threshold and inhibition dominance, which results in a larger square root factor that shifts the maximum towards the upper right and broadens it. The cumulant of the connectivity $g^{2}$ grows with $\left|J_{I} / J_{E}\right|^{2}$, which further broadens the region of the maximum [Fig. 6(b)]. The resulting asymptotic timescale [Fig. 6(c)] agrees both qualitatively and quantitatively with the intrinsic timescale [Fig. 4(d)]. This is likely due to the singleexponential shape of the autocorrelation function [Fig. 5(c)].

To investigate the interplay of the gain and the connectivity further, we vary the overall synaptic strengths by varying the excitatory weight $J_{E}$ while keeping $\left|J_{I} / J_{E}\right|$ fixed at an inhibition-dominated value [Fig. 6(d)]. Increasing $J_{E}$ in the inhibition-dominated regime shifts $\mu_{V}$ away from the effective threshold and decreases the gain; conversely $g^{2}$ grows with $J_{E}^{2}$. This interplay leads to a broad region in parameter space with an increased timescale. However, the exponential decrease of the gain is more pronounced than the quadratic increase of $g^{2}$ such that the asymptotic timescale does not continue to grow with $J_{E}$ but saturates. Thus, although increasing $J_{E}$ goes together with increased variability across neurons as in the "heterogeneous asynchronous state" described by Ostojic [62], this does not map systematically onto longer single-neuron timescales.

\section{Error function nonlinearity: external timescale}

Our theory allows arbitrary Gaussian processes as external input. To investigate the influence of an external timescale on the intrinsic timescale, we choose a zero-mean OrnsteinUhlenbeck process with

$$
C_{\mathrm{ext}}(\tau)=\frac{\sigma_{\mathrm{ext}}^{2}}{\tau_{\mathrm{m}}}\left(\frac{1}{\tau_{\mathrm{m}}}+\frac{1}{\tau_{\mathrm{ext}}}\right) e^{-|\tau| / \tau_{\mathrm{ext}}} .
$$


(a) Rate

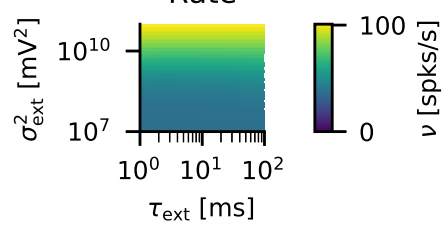

(b) Timescale

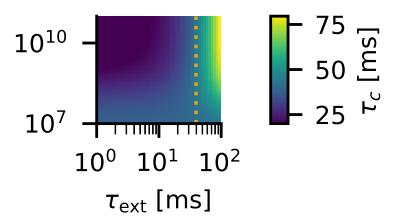

FIG. 7. Influence of colored external input. [(a) and (b)] Firing rate and intrinsic timescale for varying strength $\sigma_{\mathrm{ext}}^{2}$ and timescale $\tau_{\text {ext }}$ of an external Ornstein-Uhlenbeck process. Orange line in (b) indicates the intrinsic timescale without external input. Parameters as in Fig. 5.

Here, the scaling factors ensure that the external timescale does not influence the resulting variance of the voltage, $C_{V}(0)=\int_{-\infty}^{\infty} \tilde{\kappa}(s) C_{\mathrm{ext}}(s) d s=\sigma_{\mathrm{ext}}^{2}$ for $\tilde{\kappa}(t)=\frac{1}{2} \tau_{\mathrm{m}} e^{-|t| / \tau_{\mathrm{m}}}$.

We take the parameters from Fig. 5 where the intrinsic timescale is maximal in the absence of external input. Increasing the strength of the external input $\sigma_{\text {ext }}^{2}$ leads to an increased firing rate [Fig. 7(a)]. As desired, by construction of Eq. (29), the external timescale has a negligible effect on the firing rate at constant $\sigma_{\text {ext }}^{2}$ [Fig. 7(a)]. The effect of the external timescale on the intrinsic timescale is highly intuitive: If $\tau_{\text {ext }}$ is smaller than the intrinsic timescale without external input it decreases the intrinsic timescale, and vice versa [Fig. 7(b)]. The strength of this effect grows with the strength of the external input. In the limit of strong external input, the intrinsic timescale approaches the external timescale if $\tau_{\mathrm{ext}}>\tau_{\mathrm{m}}$; if $\tau_{\mathrm{ext}}<\tau_{\mathrm{m}}$ the intrinsic timescale approaches the minimum set by the membrane time constant.

\section{LEAKY INTEGRATE-AND-FIRE NEURONS}

Considering GLM neurons is a convenient choice due to their analytical tractability. However, their intrinsic stochasticity might fundamentally alter the network dynamics. Thus we consider the frequently used leaky integrate-and-fire neuron model in this section [24]. The synapses are taken to be current-based with an exponential time course. An analytical solution to the colored noise problem for LIF neurons is an open challenge. Here, we focus on the fluctuation-driven regime and employ an approach based on the Wiener-Rice series $[41,63,64]$ and the Stratonovich approximation thereof [40,41]. Below, we briefly introduce both the Wiener-Rice series and its Stratonovich approximation. For a comprehensive and pedagogic introduction to this approach, in particular with a focus on LIF neurons, see Ref. [65] where the approach is used to investigate LIF neurons driven by nonstationary input.

\section{A. Neuron dynamics}

The dynamics of individual neurons are governed by

$$
\begin{gathered}
\tau_{\mathrm{m}}^{\alpha} \dot{V}_{i}^{\alpha}(t)=-V_{i}^{\alpha}(t)+I_{i}^{\alpha}(t), \\
\tau_{\mathrm{s}}^{\alpha} \dot{I}_{i}^{\alpha}(t)=-I_{i}^{\alpha}(t)+\tau_{\mathrm{m}}^{\alpha} \eta_{i}^{\alpha}\left(t-d^{\alpha \beta}\right),
\end{gathered}
$$

where $V_{i}^{\alpha}$ denotes the membrane voltage, $I_{i}^{\alpha}$ the synaptic current, $\tau_{\mathrm{m} / \mathrm{s}}^{\alpha}$ the membrane/synaptic time constant, and the voltage is reset to $V_{\mathrm{r}}^{\alpha}$ and held constant during the refractory period $\tau_{\text {ref }}^{\alpha}$ whenever it reaches the threshold $\theta^{\alpha}$. Threshold crossing triggers a spike which arrives at another neuron after a delay $d^{\alpha \beta}$. We set the resting potential to zero without loss of generality and absorb the membrane resistance into the synaptic current.

\section{Effective stochastic dynamics}

The effective stochastic input with statistics governed by Eqs. (9) and (10) leads to a stochastic current with

$$
\begin{gathered}
\mu_{I}^{\alpha}=\tau_{\mathrm{m}}^{\alpha} \mu_{\eta}^{\alpha}, \\
C_{I}^{\alpha}(\tau)=\left(\frac{\tau_{\mathrm{m}}^{\alpha}}{\tau_{\mathrm{s}}^{\alpha}}\right)^{2} \int_{-\infty}^{\infty} \tilde{\kappa}^{\alpha}(\tau-s) C_{\eta}^{\alpha}(s) d s,
\end{gathered}
$$

where $\tilde{\kappa}^{\alpha}(t)=\frac{\tau_{s}^{\alpha}}{2} e^{-|t| \mid \tau_{s}^{\alpha}}$, similar to Eq. (17). Contrary to the GLM neurons, the voltage cannot become a stationary process for LIF neurons due to the fire-and-reset rule. To circumvent this problem, we use the Wiener-Rice series which relates the free process without reset to the spiking statistics.

\section{Wiener-Rice series and Stratonovich approximation}

We consider a LIF neuron after the refractory period and the voltage dynamics that results if we do not allow for another fire-and-reset. We denote this free voltage $U(t)$. Moreover, we temporarily neglect the static contribution to the input variability and drop the population index. The process starts at $U(0)=V_{\mathrm{r}}$ and produces a system of random points $\left\{t_{i}\right\}$ defined by the upcrossings $U\left(t_{i}\right)=\theta, \dot{U}\left(t_{i}\right)>0$. For this system of random points, the probability that no point falls in the interval $[0, T]$, i.e., the survival probability, is given by [40]

$S(T)=\exp \left(\sum_{s=1}^{\infty} \frac{(-1)^{s}}{s !} \int_{0}^{T} \ldots \int_{0}^{T} g_{s}\left(t_{1}, \ldots, t_{s}\right) d t_{1} \ldots d t_{s}\right)$,

where the $g_{s}\left(t_{1}, \ldots, t_{s}\right)$ are related to the free upcrossing probabilities $n_{s}\left(t_{1}, \ldots, t_{s}\right)$ calculated below, similar to the relation between moments and cumulants. For example, $g_{1}\left(t_{1}\right)=$ $n_{1}\left(t_{1}\right)$ and $g_{2}\left(t_{1}, t_{2}\right)=n_{2}\left(t_{1}, t_{2}\right)-n_{1}\left(t_{1}\right) n_{1}\left(t_{2}\right)$. Now we approximate the output process as a renewal process such that the survival probability is sufficient to describe the statistics. Instead of the survival probability, it is more convenient to consider the cumulative hazard $H(T)=-\ln S(T)$ [24], i.e.,

$$
H(T)=\sum_{s=1}^{\infty} \frac{(-1)^{s-1}}{s !} \int_{0}^{T} \cdots \int_{0}^{T} g_{s}\left(t_{1}, \ldots, t_{s}\right) d t_{1} \ldots d t_{s} .
$$

This can be regarded as a resummation of the Wiener-Rice series in terms of the $g_{s}\left(t_{1}, \ldots, t_{s}\right)$ instead of the free upcrossing probabilities $n_{s}\left(t_{1}, \ldots, t_{s}\right)[41]$.

Calculating the free upcrossing probabilities $n_{s}\left(t_{1}, \ldots, t_{s}\right)$, and thus the $g_{s}\left(t_{1}, \ldots, t_{s}\right)$, is tedious. To avoid this difficulty, Stratonovich proposed the approximation [40]

$$
H_{S}(T)=-\int_{0}^{T} n_{1}(t) \frac{\ln \left(1-\int_{0}^{T} Q\left(t, t^{\prime}\right) n_{1}\left(t^{\prime}\right) d t^{\prime}\right)}{\int_{0}^{T} Q\left(t, t^{\prime}\right) n_{1}\left(t^{\prime}\right) d t^{\prime}} d t,
$$

where $Q\left(t_{1}, t_{2}\right)=1-\frac{n_{2}\left(t_{1}, t_{2}\right)}{n_{1}\left(t_{1}\right) n_{1}\left(t_{2}\right)}$. Briefly, to derive this approximation, the $g_{s}\left(t_{1}, \ldots, t_{s}\right)$ for $s \geqslant 3$ are expressed in terms of $n_{1}\left(t_{1}\right)$ and $Q\left(t_{1}, t_{2}\right)$ such that both the symmetry of the time arguments $t_{1}, \ldots, t_{s}$ and the equal-time limit $g_{s}\left(t_{1}, \ldots, t_{1}\right)=$ 
$(-1)^{s-1}(s-1) ! n_{1}\left(t_{1}\right)^{s}$ are fulfilled; the resulting approximated $g_{s}\left(t_{1}, \ldots, t_{s}\right)$ are inserted into $H(T)$, which leads to a series that can be evaluated and yields Eq. (34). The condition $g_{s}\left(t_{1}, \ldots, t_{1}\right)=(-1)^{s-1}(s-1) ! n_{1}\left(t_{1}\right)^{s}$ holds for a system of nonapproaching points where $n_{s}\left(t_{1}, \ldots, t_{1}\right)=0$ for $s \geqslant 2$, hence Eq. (34) is an approximation constructed for such a system. Although this seems intuitively reasonable because the voltage dynamics is continuous and differentiable, this condition is violated for LIF neurons with exponential postsynaptic currents [65]. Nonetheless, it yields good results, as shown in the following.

A much simpler alternative to the Stratonovich approximation would be to set $g_{s}\left(t_{1}, \ldots, t_{s}\right)=0$ for $s \geqslant 2$, leading to $H(T)=\int_{0}^{T} n_{1}(t) d t$. This approximation is sometimes referred to as the Hertz approximation. In particular, the Hertz approximation leads to a closed expression for the hazard function $h(t) \equiv \frac{d}{d t} H(t)=n_{1}(t)$. Unfortunately, this approximation is too severe and strongly affects the resulting firing rate. The main difference between the two approximations is the asymptotic saturation of the hazard function. Thus we employ an approximation suggested by Stratonovich for long times [40]: $\int_{0}^{T} Q\left(t, t^{\prime}\right) n_{1}\left(t^{\prime}\right) d t^{\prime} \approx n_{0} \int_{0}^{\infty} Q\left(t, t^{\prime}\right) d t^{\prime} \approx$ $n_{0} \eta$ with $n_{0}=\lim _{t \rightarrow \infty} n_{1}(t)$ and $\eta=\lim _{t \rightarrow \infty} \int_{0}^{\infty} Q\left(t, t^{\prime}\right) d t^{\prime}$. Inserting this approximation into Eq. (34) leads to

$$
h_{S}(t)=\frac{\kappa_{S}}{n_{0}} n_{1}(t), \quad \kappa_{S}=-\frac{1}{\eta} \ln \left(1-n_{0} \eta\right) .
$$

Equation (35) combines the simplicity of the Hertz approximation with the asymptotic behavior of the Stratonovich approximation. The asymptotic level is given by $\lim _{t \rightarrow \infty} h_{S}(t)=$ $\kappa_{S}$; to leading order in $\eta$ we have $\kappa_{S}=n_{0}+O(\eta)$, which recovers the Hertz approximation. In the parameter regime we consider, Eq. (35) yields very similar results to Eq. (34) (see Appendix D). In all figures in the main text, we use Eq. (35). Since we approximate the output spike train as a renewal process, the hazard function Eq. (35) fully describes its statistics [24].

From the hazard function, we obtain the firing rate

$$
v^{-1}=\int_{0}^{\infty} e^{-\int_{0}^{T} h(t) d t} d T
$$

as well as the interspike-interval distribution [24]

$$
p(T)=h(T) e^{-\int_{0}^{T} h(t) d t} .
$$

From the Fourier transform of the interspike-interval distribution $\tilde{p}(f)=\int_{0}^{\infty} e^{2 \pi i f T} p(T) d T$, we obtain the spike-train power spectrum using [40]

$$
S_{x}(f)=v \frac{1-|\tilde{p}(f)|^{2}}{|1-\tilde{p}(f)|^{2}} .
$$

Thus we are left with the task of calculating $n_{1}\left(t_{1}\right)$ and $Q\left(t_{1}, t_{2}\right)$.

\section{Free upcrossing probabilities}

The free voltage dynamics are governed by Eq. (30)

$$
\tau_{\mathrm{m}} \dot{U}(t)=-U(t)+I(t),
$$

where $I$ is a Gaussian process determined by Eqs. (32) and (33), and the initial condition is $U(0)=V_{\mathrm{r}} . U$ is a nonsta- tionary Gaussian process due to the initial condition. For a sufficiently smooth Gaussian process, the upcrossing probability is given by the Kac-Rice formulas $[40,63,66]$

$$
\begin{aligned}
n_{1}(t) & =\int_{0}^{\infty} \dot{U}_{1} p\left(\theta, \dot{U}_{1} \mid V_{\mathrm{r}}, \dot{U}_{0}\right) d \dot{U}_{1}, \\
n_{2}\left(t_{1}, t_{2}\right) & =\int_{0}^{\infty} \int_{0}^{\infty} \dot{U}_{2} \dot{U}_{1} p\left(\theta, \dot{U}_{2} ; \theta, \dot{U}_{1} \mid V_{\mathrm{r}}, \dot{U}_{0}\right) d \dot{U}_{1} d \dot{U}_{2},
\end{aligned}
$$

where $p\left(\theta, \dot{U}_{1} \mid V_{\mathrm{r}}, \dot{U}_{0}\right)$ denotes the probability that the process is at the threshold after time $t$ and has velocity $\dot{U}_{1}$ given that it started at the reset at $t=0$ with velocity $\dot{U}_{0}$. Similarly, $p\left(\theta, \dot{U}_{2} ; \theta, \dot{U}_{1} \mid V_{\mathrm{r}}, \dot{U}_{0}\right)$ denotes the joint probability to be at the threshold at $t_{1}$ and $t_{2}$ with velocities $\dot{U}_{1}$ and $\dot{U}_{2}$. All integrals are over positive velocities only, because we consider upcrossings.

In both equations, we need to specify the distribution of the initial velocity $\dot{U}_{0}$. Here, it is important to take into account the biased sampling of the initial velocity [67]: at $-\tau_{\mathrm{ref}}^{\alpha}$, the neuron spiked due to an increased input current; hence, the initial velocity $\tau_{\mathrm{m}} \dot{U}_{0}=-V_{\mathrm{r}}+I_{0}$ is likely to be larger than for an $I_{0}$ drawn from the stationary current distribution. To keep the integral in Eq. (39) tractable, we assume that $I_{0}$ is Gaussian-distributed. To determine the mean and variance of this distribution, we use that the velocity of a stationary process at an upcrossing is Rayleigh-distributed [40] (details in Appendix C).

For $n_{2}\left(t_{1}, t_{2}\right)$, we consider only the stationary two-point upcrossing probability, so that it becomes a function of the time difference $t_{2}-t_{1}$ and loses the dependency on the initial velocity. After marginalizing the initial velocity in $n_{1}(t)$, we obtain

$$
\begin{gathered}
n_{1}(t)=\int_{0}^{\infty} \dot{U}_{1} p\left(\theta, \dot{U}_{1} \mid V_{\mathrm{r}}\right) d \dot{U}_{1}, \\
n_{2}\left(t_{2}-t_{1}\right)=\int_{0}^{\infty} \int_{0}^{\infty} \dot{U}_{2} \dot{U}_{1} p\left(\theta, \dot{U}_{2} ; \theta, \dot{U}_{1}\right) d \dot{U}_{1} d \dot{U}_{2},
\end{gathered}
$$

where $n_{2}(\tau)$ leads to a stationary $Q(\tau)=1-\frac{n_{2}(\tau)}{n_{0}^{2}}$. This makes the integrals in Eq. (34) considerably easier to solve numerically (details in Appendix D).

Since the free dynamics are linear, $p\left(\theta, \dot{U}_{1} \mid V_{\mathrm{r}}\right)$ and $p\left(\theta, \dot{U}_{2} ; \theta, \dot{U}_{1}\right)$ can be obtained analytically. Importantly, the integral in Eq. (39) as well as the double integral in Eq. (40) are analytically solvable using the table [59] (details in Appendixes B 2 and C). The closed-form analytical expression Eq. (B6) for the two-point upcrossing probability of a stationary Gaussian process is a novel result, to the best of our knowledge, and considerably simplifies the numerical evaluation of Eq. (35).

\section{Numerical solution of the self-consistency problem}

Just as for the GLM networks, we solve the colored noise problem using a fixed-point iteration. To initiate the algorithm, we set the rates to $v^{\alpha}=1 / \tau_{\mathrm{m}}^{\alpha}$. We use these rates to calculate the input mean, variance, and spectrum according to Eqs. (9) and (10), beginning with the diffusion approximation $S_{x}^{\alpha}(t)=v^{\alpha}$ and $\sigma_{v}^{\alpha}=0$ across neurons. Despite assuming initially equal rates across neurons, it is possible to have static input variability both due to distributed indegrees [see Eq. (8) 

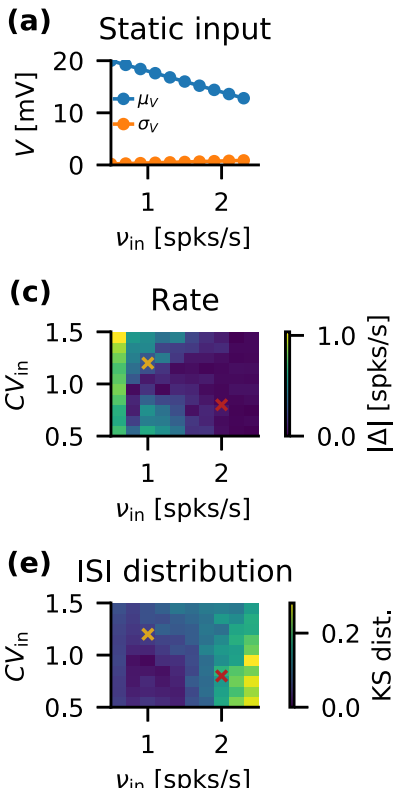

(g) ISI distribution

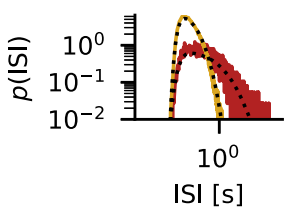

(b) Noise strength

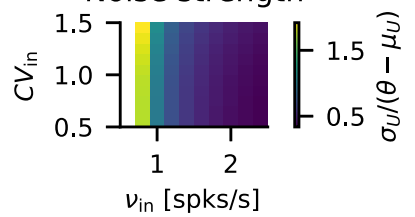

(d)

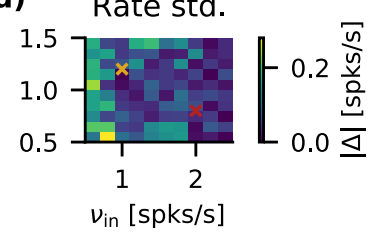

(f)

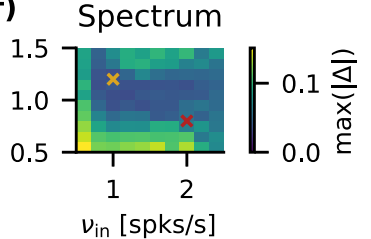

(h)

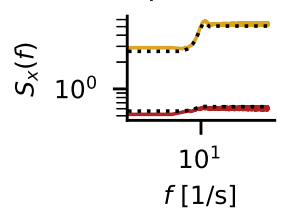

FIG. 8. Colored noise problem for LIF neurons. Comparison between theory, Eq. (35), and LIF neurons driven by Gaussian processes (GPs). (a) Mean (blue) and standard deviation across neurons (orange) of the membrane potential due to the static contribution $\tau_{\mathrm{m}} \zeta$. (b) Noise strength of the effective input measured by the standard deviation of the membrane potential fluctuations relative to the distance to threshold $\sigma_{U} /\left(\theta-\mu_{U}\right)$. (c) Absolute difference $|\Delta|$ between rate from theory and GP-driven LIF neurons. (d) Same as (c) but for the standard deviation of the rate across neurons. (e) KolmogorovSmirnov distance using 2.5-ms bins between ISI distribution from theory and GP-driven LIF neurons. (f) Maximal absolute distance $\max (|\Delta|)$ between power spectra from theory and GP-driven LIF neurons. [(g) and (h)] Example ISI distributions and power spectra from theory (black) and GP-driven LIF neurons (colored) for the parameter values indicated by crosses in (c)-(f). Parameters: $N_{E}=$ $40000, N_{I}=10000, J_{E}=0.1 \mathrm{mV},\left|J_{I} / J_{E}\right|=6.0, p=0.1, \tau_{\mathrm{m}}=$ $20 \mathrm{~ms}, \tau_{\mathrm{s}}=5 \mathrm{~ms}, \tau_{\mathrm{ref}}=2 \mathrm{~ms}, d=1.5 \mathrm{~ms}, \theta=20 \mathrm{mV}, V_{\mathrm{r}}=0 \mathrm{mV}$, and $\mu_{\mathrm{ext}}=22 \mathrm{mV}$.

and Fig. 8(a)] and due to evolution of the rates during the fixed-point iteration. To account for the static variability, we consider an ensemble of inputs $\mu^{\alpha}+\zeta^{\alpha}$ and determine the corresponding hazard functions $h_{S}^{\alpha}\left(t \mid \mu^{\alpha}+\zeta^{\alpha}\right)$, Eq. (35), output rates $v^{\alpha}\left(\mu^{\alpha}+\zeta^{\alpha}\right)$, Eq. (36), ISI distributions $p^{\alpha}\left(T \mid \mu^{\alpha}+\right.$ $\left.\zeta^{\alpha}\right)$, Eq. (37), and spectra $S_{x}^{\alpha}\left(f \mid \mu^{\alpha}+\zeta^{\alpha}\right)$, Eq. (38). From this ensemble, we obtain the final output statistics from a numerical average over the ensemble:

$$
\begin{gathered}
v^{\alpha}=\int_{-\infty}^{\infty} v^{\alpha}\left(\mu^{\alpha}+\zeta^{\alpha}\right) \mathcal{N}\left(\zeta^{\alpha} \mid 0, \sigma_{\zeta}^{\alpha}\right) d \zeta^{\alpha}, \\
\left(\sigma_{v}^{\alpha}\right)^{2}=\int_{-\infty}^{\infty}\left[v^{\alpha}\left(\mu^{\alpha}+\zeta^{\alpha}\right)-v^{\alpha}\right]^{2} \mathcal{N}\left(\zeta^{\alpha} \mid 0, \sigma_{\zeta}^{\alpha}\right) d \zeta^{\alpha},
\end{gathered}
$$

$$
\begin{aligned}
& p^{\alpha}(T)=\int_{-\infty}^{\infty} p^{\alpha}\left(T \mid \mu^{\alpha}+\zeta^{\alpha}\right) \mathcal{N}\left(\zeta^{\alpha} \mid 0, \sigma_{\zeta}^{\alpha}\right) d \zeta^{\alpha}, \\
& S_{x}^{\alpha}(f)=\int_{-\infty}^{\infty} S_{x}^{\alpha}\left(f \mid \mu^{\alpha}+\zeta^{\alpha}\right) \mathcal{N}\left(\zeta^{\alpha} \mid 0, \sigma_{\zeta}^{\alpha}\right) d \zeta^{\alpha} .
\end{aligned}
$$

We solve the above Gaussian integrals using Gauss-Hermite quadrature [60]. Gauss-Hermite quadrature of order $k$ solves Gaussian integrals of polynomials up to power $k$ exactly by construction. This allows us to keep the ensemble very small; throughout we use $k=5$. Finally, we update the statistics using incremental steps, e.g., $v_{n+1}^{\alpha}=v_{n}^{\alpha}+\varepsilon\left(\hat{v}_{n+1}^{\alpha}-v_{n}^{\alpha}\right)$ for the firing rate, where $\hat{v}_{n+1}^{\alpha}$ denotes the estimated rate based on the input at the previous step. Here, the small update step $\varepsilon<1$ is crucial because otherwise the algorithm is numerically unstable. Now we iterate and generate new input statistics. Repeated application of this scheme suggests that the self-consistent problem for the type of networks under consideration possesses only a single fixed point to which the algorithm always converges.

\section{B. Balanced random network}

First, we consider the same balanced random network as we did for the GLM neurons [Fig. 2(a)]. In particular, we place the network in the inhibition-dominated regime, drive the network with a constant external input, and use identical single-neuron parameters for excitatory and inhibitory neurons. In order to obtain a biologically plausible activity below $10 \mathrm{spks} / \mathrm{s}$, we keep the external input weak to place the network deep in the fluctuation-driven regime. In this regime, the mean input to a neuron is far below threshold and only occasional large fluctuations in the input drive it above the spike threshold [Figs. 8(a) and 8(b)]. If the mean interspike interval exceeds the correlation time of the input, the renewal approximation is admissible. Indeed, since the firing rates are low by construction, even moderate input correlation times are smaller than the inverse firing rate.

\section{Colored noise problem}

First, we isolate the colored noise problem to gauge the above approximations. To this end, we compare the theory with a population of unconnected LIF neurons driven by independent Gaussian processes (GPs). If the colored noise solution works well for isolated GP-driven LIF neurons, it will also work well for LIF neurons embedded in a balanced random network in the asynchronous irregular regime [35]. The reason for considering a population of neurons is to account for the static input variability that leads to distributed single-neuron firing rates.

We want to investigate the LIF neurons in a regime comparable to that in the balanced random network. However, we do not determine the effective input statistics using network simulation results here, because this would preclude a systematic scan over the parameters of the input, which consists of both external and recurrent network contributions. Instead, we fix the effective external input and determine the statistics of the effective recurrent input in terms of the input spiking statistics 
$v_{\text {in }}, \sigma_{v}^{\text {in }}=0$ across neurons, and

$$
S_{x}^{\text {in }}(f)=v_{\text {in }} \frac{1-\left|\left(1-2 \pi i \mathrm{CV}_{\text {in }}^{2} f / \nu_{\text {in }}\right)^{-1 / \mathrm{CV}_{\text {in }}^{2}}\right|^{2}}{\left|1-\left(1-2 \pi i \mathrm{CV}_{\mathrm{in}}^{2} f / \nu_{\text {in }}\right)^{-1 / \mathrm{CV}_{\mathrm{in}}^{2}}\right|^{2}},
$$

corresponding to a gamma process with rate $v_{\text {in }}$ and $\mathrm{CV}$ of the ISI distribution $\mathrm{CV}_{\text {in }}$, cf. Eq. (38). This leaves a twodimensional parameter space spanned by $v_{\text {in }}$ and $\mathrm{CV}_{\text {in }}$. From the spiking statistics, we obtain the statistics of the effective input using Eqs. (9) and (10) where $\bar{g}$ and $g$ are determined by the parameters of the balanced random network. Note that although $\sigma_{v}^{\text {in }}=0$, the static variability of the effective input is nonzero, $\sigma_{\zeta}>0$, due to the distributed indegree, see Eq. (8) and Fig. 8(a). Hence, we can compare both the averaged output statistics and the rate variability in the population. For the comparison, we simulate 250 GP-driven LIF neurons for $50 \mathrm{~s}$ with a time step of $0.05 \mathrm{~ms}$; we use the same interval and time step for the theory.

Guided by the regime attained in full simulations, we choose $v_{\text {in }} \in[0.5,2.5] \mathrm{spks} / \mathrm{s}$ and $\mathrm{CV}_{\text {in }} \in[0.5,1.5]$ (Fig. 8). The network is in the inhibition-dominated regime; thus the mean input decreases with $v_{\text {in }}$ starting from the value that brings the membrane potential on average to threshold [Fig. 8(a)]. In contrast, the static variability increases monotonically with $v_{\text {in }}$ [Fig. 8(a)]. To measure the strength of the dynamic variability, we divide the resulting standard deviation of the free membrane voltage by the distance of the mean free membrane voltage to the threshold, $\sigma_{U} /\left(\theta-\mu_{U}\right)$. Since the numerator grows with $\sqrt{\nu_{\text {in }}}$ while the denominator grows linearly with $v_{\text {in }}$ in inhibition-dominated networks, the standard deviation relative to the distance to threshold decreases with increasing $v_{\text {in }}$; in contrast, it slightly increases with increasing $\mathrm{CV}_{\text {in }}$ [Fig. 8(b)]. For the entire parameter regime, the absolute difference in the firing rate is smaller than $1 \mathrm{spks} / \mathrm{s}$ and it is maximal at the brink of the fluctuation-driven regime [Fig. 8(c)]. For the static rate variability, we also consider the absolute difference, which is below $0.3 \mathrm{spks} / \mathrm{s}$ throughout the parameter space [Fig. 8(d)]. Next, we compare the ISI distributions using their Kolmogorov-Smirnov distance, i.e., the maximal absolute difference between the cumulative distributions. The Kolmogorov-Smirnov distance is maximal deep in the fluctuation-driven regime where the firing rate is well below $1 \mathrm{spks} / \mathrm{s}$ and the estimate of the ISI distribution is noisy [Fig. 8(e)]. Finally, we compare the output spectra using the maximum absolute distance between the scaled spectra $S_{x}(f) / v$. Here, the deviation is below 0.1 in most parts of the parameter space except for low $\mathrm{CV}_{\text {in }} \leqslant 0.6$, high $\mathrm{CV}_{\text {in }} \geqslant 1.3$, and at the brink of the fluctuation-driven regime [Fig. 8(f)]. To give meaning to the quantitative results, we plot two example ISI distributions [Fig. 8(g)] and spectra [Fig. 8(h)]. For the ISI distribution, we see the noisy estimate at low rates. For the spectra, we note that the main difference is a constant offset caused by a small error in the rate, see Eq. (38), while the shape is well matched.

To conclude, the above approximations work well in the fluctuation-driven regime for moderate values $0.6<\mathrm{CV}_{\text {in }}<1.3$. Within this regime, the firing rate and its variability across neurons, the ISI distribution, and the power spectra are well predicted. Most importantly for the prediction
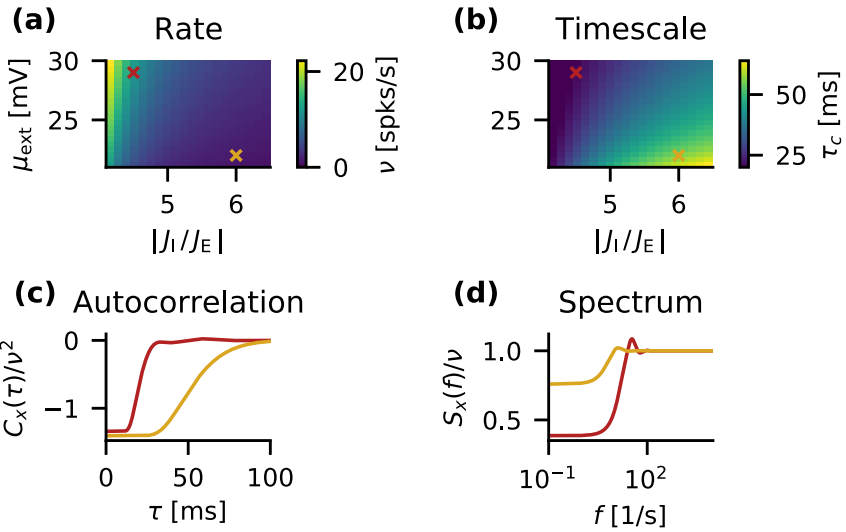

FIG. 9. Parameter scan for a balanced random network of LIF neurons using Eq. (35). [(a) and (b)] Firing rate and intrinsic timescale for varying external input $\mu_{\mathrm{ext}}$ and relative inhibitory strength $\left|J_{I} / J_{E}\right|$. [(c) and (d)] Scaled autocorrelation $C_{x}(\tau) / v^{2}$ and power spectrum $S_{x}(f) / v$ for the parameter values indicated by symbols in (a,b). Further parameters as in Fig. 8.

of the intrinsic timescale, the theory closely predicts the scaled spectrum $S_{x}(f) / \nu$.

\section{Timescales in balanced random networks of LIF neurons}

Having established the validity of the theory, we employ it to investigate the intrinsic timescale. It is well known that increasing the overall synaptic strength leads to a network state with long temporal correlations [37,62]. However, this state comes along with giant fluctuations of the membrane potential [68] which are well beyond the physiological regime and which our theory can capture only to a limited extent (in particular, it underestimates the strong increase in low-frequency power observed for strong couplings [37,69]). Hence, we focus on the influence of the external input $\mu_{\text {ext }}$ and the inhibition dominance $\left|J_{I} / J_{E}\right|$, in line with our above investigations for GLM neurons. We solve the theory on a $\Delta t=0.05 \mathrm{~ms}$ grid to a maximum of $T=10 \mathrm{~s}$, use an ensemble size of $k=5$ for the Gauss-Hermite quadrature, and choose an update step $\varepsilon=0.2$.

We investigate the regime $\left|J_{I} / J_{E}\right| \in[4.1,6]$ and $\mu_{\mathrm{ext}} \in[21,30] \mathrm{mV}$. Within this regime, the rate is below approximately $20 \mathrm{spks} / \mathrm{s}$, increases with $\mu_{\text {ext }}$, and decreases with $\left|J_{I} / J_{E}\right|$ [Fig. 9(a)]. In contrast, the intrinsic timescale decreases with $\mu_{\text {ext }}$, increases with $\left|J_{I} / J_{E}\right|$, and reaches a maximum of approximately $60 \mathrm{~ms}=3 \tau_{\mathrm{m}}$ [Fig. 9(b)]. The autocorrelation function reveals that the nature of these longer intrinsic timescales in LIF networks is fundamentally different to the GLM networks above [Fig. 9(c)]: in the GLM networks, the autocorrelation function is positive, which corresponds to an increased probability to spike in succession; in the LIF networks it is negative, which corresponds to a prolonged effective refractory period caused by the fire-and-reset mechanism in combination with the input statistics. Indeed, in the corresponding power spectra and their zero-frequency limit, we see that the $\mathrm{CV}$ is well below 1 [Fig. 9(d)]. Hence, the process is more regular than a Poisson process, as opposed to the high irregularity $\mathrm{CV}>1$ that would go along with bursty spiking. 
(a)

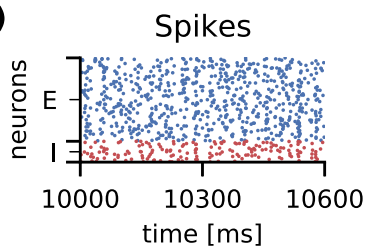

(c)

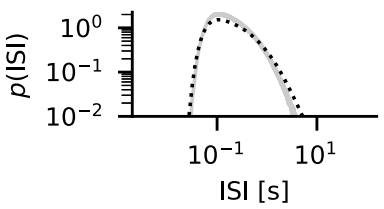

(e)

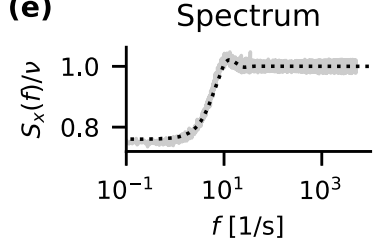

(b) Rate distribution

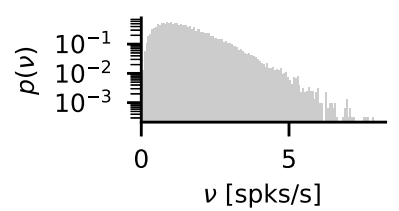

(d) Autocorrelation

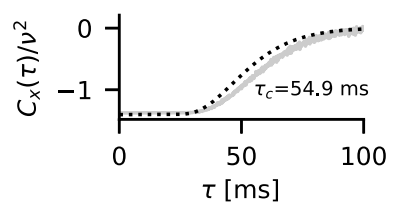

(f) Population spectrum

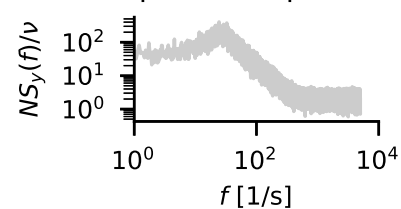

FIG. 10. Balanced random network of LIF neurons. (a) Raster plot of $2 \%$ of the excitatory (blue) and inhibitory (red) neurons. (b) Firing rate distribution across all neurons. [(c)-(e)] Population-averaged ISI distribution, population-averaged autocorrelation function, and population-averaged power spectrum from simulation (gray) and theory (black). (f) Power spectrum of the population activity. Parameters as in Fig. 8.

\section{Simulation of balanced random network of LIF neurons}

We validate the theoretical predictions for the balanced random network of LIF neurons by comparing with a network simulation. To acquire sufficient statistics, we simulate the network for $T=2.5 \mathrm{~min}$ with time step $\Delta t=0.1 \mathrm{~ms}$ and discard the first $10 \mathrm{~s}$ as an initial transient. After this transient, the network is in an asynchronous irregular state [Fig. 10(a)]. The rates of individual neurons are mostly below $5 \mathrm{spks} / \mathrm{s}$ with a peak at around $1 \mathrm{spks} / \mathrm{s}$ [Fig. 10(b)]. The theory closely predicts the ISI distribution apart from a slight overestimation of the tail [Fig. 10(c)]. Thus the resulting autocorrelation function is also well matched and the predicted intrinsic timescale of approximately $55 \mathrm{~ms}$ is confirmed [Fig. 10(d)]. Also the scaled spectrum is closely reproduced and reveals a $\mathrm{CV}^{2} \approx$ 0.75 [Fig. 10(e)].

To illustrate the difference between the single-unit and the population statistics, we furthermore plot the power spectrum of the population activity $y(t)=\frac{1}{N} \sum_{i=1}^{N} x_{i}(t)$ [Fig. 10(f)]. For vanishing cross-correlations, these two spectra would be proportional to each other. Already weak cross-correlations can shape the population spectrum since their contribution is of $O\left(N^{2}\right)$ compared to $O(N)$ contributions from the autocorrelations, leading to the clear differences we see between the single-unit and the population spectrum. A notable difference between the two spectra is the peak around $30 \mathrm{~Hz}$ in the population spectrum, contrasting with the roughly $10-\mathrm{Hz}$ peak in the single-unit spectrum. Furthermore, the population spectrum displays increased power at low frequencies compared to high frequencies, while the reverse is true for the single-unit spectrum.

\section{Biologically constrained network model}

Thus far, we only considered balanced random networks with identical excitatory and inhibitory neurons that reduce to a single effective population. Despite this simplification, these balanced random networks already span a large parameter space. Here, we apply our theory to a multipopulation network model constrained by biological data [43]. Beyond the aspect of multiple populations, this network model allows us to highlight two additional features of our theory that we left out thus far: the possibility to include external Poisson input and distributed synaptic weights. We solve the theory on a $\Delta t=0.05 \mathrm{~ms}$ grid to a maximum of $T=10 \mathrm{~s}$, use an ensemble size of $k=5$ for the Gauss-Hermite quadrature, choose an update step $\varepsilon=0.1$, and initialize all populations with a rate of $10 \mathrm{spks} / \mathrm{s}$.

The model represents the neurons under $1 \mathrm{~mm}^{2}$ of surface of generic early sensory cortex. It comprises eight populations: layers $2 / 3,4,5$, and 6 with a population of excitatory cells and inhibitory interneurons for each layer [Fig. 11(a)]. In total, this leads to 77169 neurons connected via approximately $3 \times 10^{8}$ synapses, with population-specific connection probabilities $p^{\alpha \beta}$ based on an extensive survey of the anatomical and physiological literature. In contrast to the original model, we directly use the connection probabilities to create the connectivity such that the total number of synapses can vary across instantiations of the model, and we draw source and target neurons without replacement, so that multapses are not allowed. Transmission delays follow truncated normal distributions with mean \pm standard deviation of $1.5 \pm 0.75 \mathrm{~ms}$ for excitatory source neurons and $0.75 \pm 0.375 \mathrm{~ms}$ for inhibitory source neurons, both with a cutoff at $0.1 \mathrm{~ms}$. The synaptic strengths $J_{i j}^{\alpha \beta}$ are normally distributed with $\mu_{J}^{\alpha I}=-351.2 \mathrm{pA}$ for inhibitory source neurons and $\mu_{J}^{\alpha E}=87.8 \mathrm{pA}$ for excitatory source neurons except for connections from layer 4 excitatory to layer $2 / 3$ excitatory neurons, which have a mean strength of $175.6 \mathrm{pA}$. For all synaptic strengths, the standard deviation is fixed to $10 \%$ of the mean. The network is driven by external Poisson input with layer-specific rates (for further details see Ref. [43]).

The intrinsic parameters of the neurons do not vary across populations. Shaped by the connectivity, a layer-specific activity arises [Fig. 11(b)] with mean firing rates between 1 and $10 \mathrm{spks} / \mathrm{s}$ [Fig. 11(c)] and a standard deviation across neurons between 1 and 5 spks/s [Fig. 11(d)]. While the quantitative agreement is not perfect, our theory captures the specificity of both mean firing rate and its variability across neurons well.

A prominent feature of the model are oscillations on the population level [70] which are already visible in the raster plot of only $2 \%$ of the population [Fig. 11(b)]. These oscillations lead to a clear peak at about $80 \mathrm{~Hz}$ in the power spectrum of the population activity in all layers [70]. Here, we only show a representative population spectrum [Fig. 11(e)]. These population-level oscillations clearly violate the independence assumption of the effective inputs. Thus they could potentially explain the deviations of the predicted firing rate from the simulation.

For most populations, the peak in the population-level oscillations also manifests itself in the population-averaged single-unit spectra [Figs. 11(f) and $11(\mathrm{~g})$ ]. Apart from this 
(a)

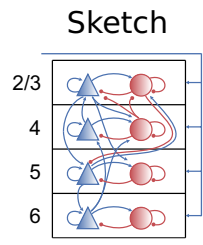

(b)

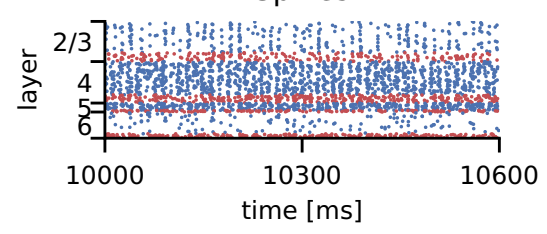

(c) Rate (mean)
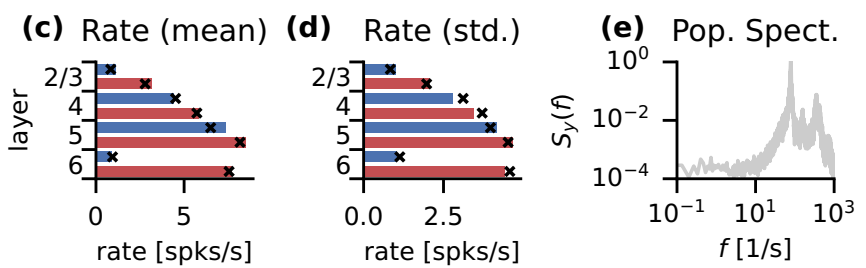

(f)

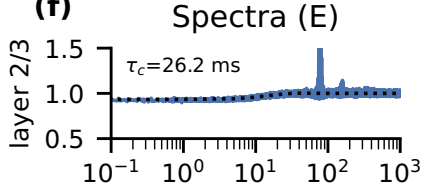

(g)
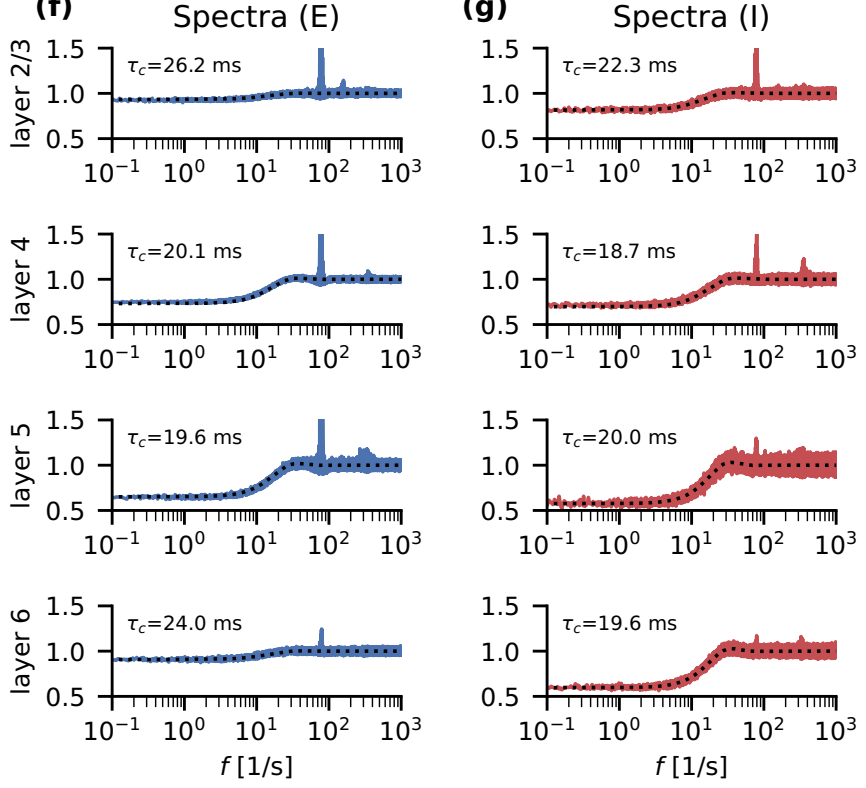

FIG. 11. Multipopulation network of LIF neurons. (a) Sketch of the model, figure adapted from [43]. (b) Raster plot of $2 \%$ of the neurons of each population. [(c) and (d)] Neuron-averaged firing rates and their standard deviation from simulations (bars) and theoretical predictions (black crosses). (e) Population spectrum of the layer 4 excitatory population. [(f) and (g)] Spike-train power spectra obtained from simulations (colored) and theory (black) and the corresponding intrinsic timescale. Parameters as specified in [43].

peak, our theory closely captures the shape of all spectra [Figs. 8(f) and 8(g)]. Note that, despite the large heterogeneity of mean rates, the intrinsic timescale is similar across populations. As in the balanced random network, the intrinsic timescales are on the order of magnitude of the membrane time constant (here $10 \mathrm{~ms}$ ); concretely, the intrinsic timescale is approximately twice as large.

\section{DISCUSSION}

We developed a self-consistent theory for the second-order statistics, in particular the intrinsic timescales as defined by autocorrelation decay times, in block-structured random networks of spiking neurons in an asynchronous irregular state. Orthogonal to approaches based on the mean activity of a population of neurons, we consider population-averaged single-neuron statistics. To this end, we built on the modelindependent dynamic mean-field theory (DMFT) developed in Ref. [30] and applied it to networks of spiking neurons. We sketched the derivation starting from the characteristic functional of the recurrent input, Eq. (3), to expose the inherent assumptions of the DMFT as well as its main result. In particular, we showed that the mean-field equations, Eqs. (9) and (10), where the connectivity matrix enters only through its first two cumulants, account for both (static) interneuron variability and (dynamic) temporal fluctuations. In order to close the self-consistency problem, we derived a novel analytical solution for the output statistics of a generalized linear model (GLM) neuron with error-function nonlinearity driven by a Gaussian process (GP), Eq. (25), and an analytical approximation for the output statistics of a GP-driven leaky integrate-and-fire (LIF) neuron in the fluctuation-driven regime, Eq. (35). These theoretical results yield firing rate distributions, spike-train power spectra, and interspike interval distributions that are close to those obtained from numerical simulations (Figs. 2, 5, and 10) even for a complex, biologically constrained network model (Fig. 11).

The excellent agreement between theory and simulations demonstrates the validity of the DMFT approximation, i.e., the approximation of the recurrent inputs as independent Gaussian processes. The validity of the DMFT approximation is most clearly demonstrated by the networks of GLM neurons, since in that case the DMFT assumption constitutes the only approximation, while the remainder of the solution is exact; while for the LIF networks, additional approximations are made, so that the effects of the DMFT assumption can be less well isolated.

Focusing on balanced random networks, we leveraged our theory to investigate the influence of network parameters on the intrinsic timescale for both GLM (Figs. 3 and 4) and LIF (Fig. 9) neurons. For the former neuron model with error function nonlinearity, our theory unveils that a product of two factors determines the intrinsic timescale [Eq. (27), Fig. 6]: the gain of the rate autocorrelation function with respect to changes in the membrane voltage autocorrelation function for $\tau \rightarrow \infty$, Eq. (28), and the variance of the connectivity, Eq. (6). Furthermore, providing a temporally correlated external drive causes the intrinsic timescale to monotonically approach the extrinsic timescale as the input strength is increased (Fig. 7).

For both GLM neurons with error function nonlinearity and LIF neurons, we find parameter regimes where the intrinsic timescale $\tau_{c}$ is longer than the largest time constant of the single-neuron dynamics, the membrane time constant $\tau_{\mathrm{m}}$ (Figs. 5 and 9). This demonstrates that the recurrent dynamics shape the intrinsic timescale. Note that we consider a regime where the inverse firing rate $v^{-1}$ is large compared to $\tau_{\mathrm{m}}$. In contrast, $[18,29]$ consider the opposite regime where slow neuronal timescales lead to effective rate dynamics, and the spiking noise is either left out or treated perturbatively. Our results show that it is possible to obtain longer intrinsic timescales even in a regime where the white component of the spiking noise contributes non-negligibly to the membrane voltage fluctuations. However, the temporal structure that causes the prolonged intrinsic timescale is very different for the two models that we consider: For GLM neurons, the 
autocorrelation is positive for a period on the order of $\tau_{c}$, corresponding to an increased spiking probability. For LIF neurons, the autocorrelation function is negative, corresponding to a prolonged effective refractory period.

Furthermore, LIF networks exhibit a minimum in the intrinsic timescale [37], while the corresponding GLM networks exhibit a maximum (Fig. 6). We hypothesize that this difference is due to the difference in the temporal structure: The minimum in the timescale for LIF networks is caused by a switch from an increased effective refractory period (a negative autocorrelation function for $\tau \rightarrow 0$ ) to an increased probability for another spike (a positive autocorrelation function for $\tau \rightarrow 0$ ). This hypothesis is consistent with the switch from decreased low-frequency power, $S_{x}(f \rightarrow 0)<v$, to increased low-frequency power, $S_{x}(f \rightarrow 0)>v$, highlighted in Refs. [37,69]. For GLM networks, this switch and hence the minimum is absent. Instead, the more subtle interplay between the gain and the variance of the connectivity leads to the maximum. The presence of a maximum rather than a minimum in the intrinsic timescales renders the GLM networks more similar to networks of rate units [15]. If similar mechanisms are at play as in rate networks, the white spiking noise of the input to the GLM neurons may temper the size of the largest possible timescale [21]. However, due to the inherent stochasticity of GLM neurons, it is unclear whether the maximum occurs at a transition to chaos as it does in rate networks [15].

Considering a more complex block-structured network model that is constrained by biological data [43] exposes limits of our theory: while the theory accurately captures the nonoscillatory components of the power spectra, it misses a high-frequency oscillation (Fig. 11). These high-frequency oscillations are caused by correlated activity on the population level [70]; hence, the peak in the population-averaged singleneuron spectra demonstrates an interplay between single-unit and population-level statistics that was absent in the simpler balanced random network models. By construction, our theory only accounts for population-averaged single-neuron statistics and thus misses the high-frequency peak. It is an interesting challenge to derive a self-consistent theory on both scales simultaneously.

In general, the limits of DMFT when applied to spiking networks merit further investigation. For example, assuming that the network is sparse, $K \ll N$ or $p \ll 1$, is not a necessary condition for a DMFT to apply [18]. Nonetheless, increasing sparsity reduces the pairwise correlations between the neurons $[26,62,71]$ such that DMFT is expected to yield better results. Another important aspect is that for the synaptic weights scaling as $J_{i j}=O(1 / \sqrt{K})$, the fluctuations of the mean input $\mu_{\eta}(t)$ can be $O(1)$, i.e., not scale with $K^{-\alpha}, \alpha>0$, as the network size increases and $p$ is kept constant. In Eq. (7), $\mu_{\eta}(t)$ and $C_{\eta}\left(t, t^{\prime}\right)$ are replaced by their average, neglecting fluctuations; including the fluctuations of the mean input would lead to an additional term in $C_{\eta}\left(t, t^{\prime}\right)$ [28]. Since these fluctuations of the mean input reflect pairwise correlations, the latter need to be small for the theory to be accurate. The above scaling argument shows that it is nontrivial that the pairwise correlations vanish, even in the large network limit. They only do so for an asynchronous state in which the pairwise correlations are small already for finite networks, e.g., due to a sparse network or due to inhibitory feedback [27]. Conversely, if a network is in an asynchronous irregular state, which has low pairwise correlations by definition, DMFT is expected to yield reliable results.

The heterogeneity of timescales even within a cortical area [72] suggests another interesting extension, namely to calculate the variability of the timescale within a population. This requires calculating the variability of the second-order statistics, which has recently been achieved for linear rate networks [73] but to the best of our knowledge is an open challenge even for simple nonlinear rate networks, let alone for spiking networks.

The microscopic theory presented here enables direct comparisons with experimental measurements of neuronlevel intrinsic timescales [2], in contrast to previous works which have considered population rate models [10,74]. It is important to distinguish between neuron-level and population-level autocorrelations, since the latter are shaped by $O\left(N^{2}\right)$ cross-correlations and can therefore differ substantially from neuron-level autocorrelations, as we have illustrated for the balanced random network model [Figs. 10(e) and 10(f)] and the biologically constrained network model [Figs. 11(e)-11(g)].

Establishing a direct link between the connectivity and the emergent intrinsic timescales opens up the possibility of a thorough investigation of the effect of network architecture. Moreover, within our theory, it is possible to account for population-specific intrinsic neuron parameters. Thus the theory also provides an avenue for investigations of the complex interplay between intrinsic parameters [8,9] and the network structure [10]. In this context, an interesting application is clustered networks which feature slow switching between transiently active clusters [75]. In particular, clustered networks with both excitatory and inhibitory clusters [76-78] could be of interest because they robustly give rise to winnerless competition. From a modeler's point of view, uncovering mechanisms shaping intrinsic timescales could be used to fine-tune network models [79-82] to match the experimentally observed hierarchy of timescales [2]. Focusing on computational aspects, diverse timescales strongly enhance the computational capacity of a recurrent network [83-85], and neurons with long intrinsic timescales carry more information in a working memory task [86] (but see [87]). In this light, the results presented here may also contribute to improved understanding of aspects of information processing in the brain.

\section{ACKNOWLEDGMENTS}

This work was supported by the European Union's Horizon 2020 Framework Programme for Research and Innovation under Specific Grants Agreement No. 785907 and No. 945539 (Human Brain Project SGA2, SGA3), the Jülich-Aachen Research Alliance (JARA), and DFG Priority Program "Computational Connectomics” (SPP 2041; Project 347572269). A.v.M. would like to thank Moritz Helias for many helpful and inspiring discussions about dynamical mean-field theory and spiking neurons. We further thank Tilo Schwalger for his insights about the Stratonovich approximation and for sharing his unpublished manuscript, and Jasper Albers, Anno Kurth, Alessandra Stella, Christian Keup, David Dahmen, and 
the anonymous reviewers for valuable comments on an early version of the manuscript.

\section{APPENDIX A: CHARACTERISTIC FUNCTIONALS}

Here, we briefly introduce the characteristic functionals for both types of stochastic processes we consider: Gaussian processes and point processes. We closely follow Stratonovich's book [40], in particular Chaps. I.1. and I.6.

\section{Stochastic processes}

The characteristic functional of a stochastic process $\xi(t)$ is defined as

$$
\Phi_{\xi}[u(t)]=\left\langle e^{i \int_{0}^{T} u(t) \xi(t) d t}\right\rangle_{\xi}
$$

where $u(t)$ is an arbitrary test function. In terms of the cumulants $k_{r}\left(t_{1}, \ldots, t_{r}\right)$ the characteristic functional can be written as

$$
\Phi_{\xi}[u(t)]=e^{\sum_{s=1}^{\infty} \frac{i^{s}}{s !} \int_{0}^{T} \ldots \int_{0}^{T} k_{s}\left(t_{1}, \ldots, t_{s}\right) u\left(t_{1}\right) \ldots u\left(t_{s}\right) d t_{1} \ldots d t_{s}} .
$$

All properties of a stochastic process are determined by its characteristic functional.

If all cumulants except for the first vanish, the process is deterministic and has the characteristic functional

$$
\Phi_{\xi}[u(t)]=\left\langle e^{i \int_{0}^{T} u(t) \xi(t) d t}\right\rangle_{\xi}=e^{i \int_{0}^{T} u(t) \xi(t) d t} .
$$

In this case, the first cumulant coincides with the process itself, $k_{1}(t)=\xi(t)$. If only the first and the second cumulants are nonvanishing, the process is a Gaussian process. The corresponding characteristic functional reads

$$
\Phi_{\xi}[u(t)]=e^{i \int k_{1}\left(t_{1}\right) u\left(t_{1}\right) d t_{1}-\frac{1}{2} \iint u\left(t_{1}\right) k_{2}\left(t_{1}, t_{2}\right) u\left(t_{2}\right) d t_{1} d t_{2}} .
$$

If the Gaussian process is stationary, $k_{1}\left(t_{1}\right)=k_{1}$ and $k_{2}\left(t_{1}, t_{2}\right)=k_{2}\left(t_{2}-t_{1}\right)$, the characteristic functional simplifies further to $\Phi_{\xi}[u(t)]=e^{i k_{1} \int u\left(t_{1}\right) d t_{1}-\frac{1}{2} \iint u\left(t_{1}\right) k_{2}\left(t_{2}-t_{1}\right) u\left(t_{2}\right) d t_{1} d t_{2}}$.

The characteristic functional describes the statistics at all points in time. It is often useful to relate the characteristic functional to the distribution of the values of $\xi(t)$ at fixed points in time, for instance to compute the statistics of the current at upcrossings and after the refractory period, or to obtain marginal activity statistics which, given stationarity, reflect time-averaged activity. To this end, we can use the test functions $u(t)=u_{1} \delta\left(t-t_{1}\right)$ and $u(t)=u_{1} \delta\left(t-t_{1}\right)+u_{2} \delta\left(t-t_{2}\right)$ to obtain

$$
\begin{gathered}
\Phi_{\xi}\left(u_{1}\right)=e^{i k_{1}\left(t_{1}\right) u_{1}-\frac{1}{2} k_{2}\left(t_{1}, t_{1}\right) u_{1}^{2}}, \\
\Phi_{\xi}\left(u_{1}, u_{2}\right)= \\
e^{i\left(k_{1}\left(t_{1}\right) u_{1}+k_{1}\left(t_{2}\right) u_{2}\right)} \\
\times e^{-\frac{1}{2}\left(k_{2}\left(t_{1}, t_{1}\right) u_{1}^{2}+2 k_{2}\left(t_{1}, t_{2}\right) u_{1} u_{2}+k_{2}\left(t_{2}, t_{2}\right) u_{2}^{2}\right)} .
\end{gathered}
$$

These are the characteristic functions of a Gaussian with cumulants determined by $k_{1}$ and $k_{2}$. Knowing these characteristic functions for all times $t_{1}$ and $t_{2}$ provides the full picture; this is the marginalization property of Gaussian processes [88].

\section{Point processes}

The equivalence to the characteristic functional for a point process is the generating functional. For a spike train $\left\{t_{1}, \ldots, t_{n}\right\}$ (a "system of random points" in Stratonovich's naming) with $t_{i} \in[0, T]$ for all $i$, the generating functional is defined by

$$
L_{T}[v(t)]=\left\langle\prod_{j=1}^{n}\left[1+v\left(t_{j}\right)\right]\right\rangle
$$

Here, the number of spikes $n$ is itself a random variable because the average is taken with respect to all possible realizations of the spike train [89].

For point processes, the role of the moments is taken by the "distribution functions" $n_{r}\left(t_{1}, \ldots, t_{r}\right)$ which denote the probability of having at least one point in each interval $\left[t_{i}, t_{i}+d t\right]$. The role of the cumulants is taken by the functions $g_{r}\left(t_{1}, \ldots, t_{r}\right)$, which are related to the distribution functions as the cumulants of a stochastic process are related to its moments. In terms of the $g_{r}\left(t_{1}, \ldots, t_{r}\right)$, the generating functional can be written as [89]

$$
L_{T}[v(t)]=e^{\sum_{s=1}^{\infty} \frac{1}{s !} \int_{0}^{T} \ldots \int_{0}^{T} g_{s}\left(t_{1}, \ldots, t_{s}\right) v\left(t_{1}\right) \ldots v\left(t_{s}\right) d t_{1} \ldots d t_{s}} .
$$

The generating functional is directly related to a few useful quantities: The characteristic function of the number of spikes $n$ in the interval $[0, T]$ is given by $\left\langle e^{i n u}\right\rangle=L_{T}\left[e^{i u}-1\right]$; the probability that no point falls into $[0, T]$, i.e., the survival probability, is given by $L_{T}[-1]$. The simplest case of a point process where only $g_{1}$ is nonvanishing is a Poisson process. The corresponding generating functional reads

$$
L_{T}[v(t)]=\exp \left(\int_{0}^{T} g_{1}\left(t_{1}\right) v\left(t_{1}\right) d t_{1}\right)
$$

with survival probability $S(T)=L_{T}[-1]=e^{-\int_{0}^{T} g_{1}\left(t_{1}\right) d t_{1}}$.

The generating functional is directly related to the characteristic functional of the stochastic process $\xi(t)=\sum_{j=1}^{n} \delta(t-$ $\left.t_{j}\right)$ :

$$
\Phi_{\xi}[u(t)]=\left\langle e^{i \sum_{j=1}^{n} u\left(t_{j}\right)}\right\rangle=L_{T}\left[e^{i u(t)}-1\right] .
$$

This relation links the distribution functions $n_{r}$ through the $g_{r}$ to the cumulants of the spike train. For example, the characteristic functional of a Poisson spike train is

$$
\Phi_{\xi}[u(t)]=\exp \left(\int_{0}^{T} g_{1}\left(t_{1}\right)\left(e^{i u\left(t_{1}\right)}-1\right) d t_{1}\right) .
$$

Note that by convention, $g_{1}(t)$ is typically called $\lambda(t)$ for a Poisson process-we adopted this convention in the main text, in particular in Eq. (14). Expanding the exponent on the righthand side of Eq. (A7) to second order in $u(t)$, we obtain the relations

$$
\begin{aligned}
k_{1}\left(t_{1}\right) & =g_{1}\left(t_{1}\right), \\
k_{2}\left(t_{1}, t_{2}\right) & =g_{1}\left(t_{1}\right) \delta\left(t_{1}-t_{2}\right)+g_{2}\left(t_{1}, t_{2}\right)
\end{aligned}
$$

between the $g_{r}$ and the first two cumulants of the spike train. 


\section{Gaussian integrals}

We solve several Gaussian integrals using the impressive table by Owen [59]. First, we introduce his notation

$$
G(x)=\frac{1}{2}(1+\operatorname{erf}(x / \sqrt{2})), \quad g(x)=\frac{1}{\sqrt{2 \pi}} e^{-x^{2} / 2}
$$

for the standard normal CDF and PDF. Furthermore, we need Owen's $T$ function

$$
T(h, a)=\frac{1}{2 \pi} \int_{0}^{a} \frac{e^{-\frac{1}{2} h^{2}\left(1+x^{2}\right)}}{1+x^{2}} d x .
$$

All formulas were numerically validated using numerical integration routines implemented in SCIPY [90].

\section{a. GLM error function}

Here, we derive Eqs. (24) and (25). In the notation of Eq. (23), we have $\phi(x)=G(x)$.

For the mean, we need the expectation $\langle\phi(z)\rangle$ where $z$ is Gaussian with mean $\mu$ and variance $\sigma^{2}$. Equivalently, we can calculate $\langle\phi(\mu+\sigma x)\rangle$ where $x$ is standard normal. Expressing the standard normal Gaussian expectations using $g(x)$, we have

$$
\langle\phi\rangle=\int_{-\infty}^{\infty} g(x) G(\mu+\sigma x) d x .
$$

Using Eq. (10,010.8) from Ref. [59], we get

$$
\langle\phi\rangle=G\left(\frac{\mu}{\sqrt{1+\sigma^{2}}}\right) .
$$

Equation (24) follows after taking the multiplying factor $c_{1}$ and $\mu=\mu_{V}-\theta$ from Eq. (14) into account.

For the second moment, we need $\left\langle\phi\left(z_{1}\right) \phi\left(z_{2}\right)\right\rangle$ were $z_{1}$ and $z_{2}$ are jointly Gaussian with mean $\mu$, variance $\sigma^{2}$ and correlation coefficient $\rho$. Equivalently, we can calculate $\langle\phi(\mu+$ $\beta x-\alpha y) \phi(\mu+\beta x+\alpha y)\rangle$ where $x$ and $y$ are standard normal and $\alpha=\sigma \sqrt{(1-\rho) / 2}, \beta=\sigma \sqrt{(1+\rho) / 2}$. Again using $g(x)$ to express the standard normal Gaussian expectations, we get

$$
\begin{aligned}
\langle\phi \phi\rangle & =\int_{-\infty}^{\infty} g(x) I(x) d x \text { with } \\
I(x) & =\int_{-\infty}^{\infty} g(y) G(\mu+\beta x-\alpha y) G(\mu+\beta x+\alpha y) d y .
\end{aligned}
$$

Now, we use Eq. $(20,010.3)$ in Ref. [59] for $I(x)$ to obtain

$$
\langle\phi \phi\rangle=\int_{-\infty}^{\infty} g(x)(G(a+b x)-2 T(a+b x, c)) d x
$$

with $\quad a=\mu / \sqrt{1+\sigma^{2}(1-\rho) / 2}, \quad b=$ $\sigma \sqrt{1+\rho} / \sqrt{2+\sigma^{2}(1-\rho)}, \quad c=\sqrt{1+\sigma^{2}(1-\rho)}, \quad$ and Owen's T function $T(h, a)$. For the final integral, we use Eqs. (10,010.8) and (c00,010.1) from Ref. [59] to derive

$$
\langle\phi \phi\rangle=G\left(\frac{\mu}{\sqrt{1+\sigma^{2}}}\right)-2 T\left(\frac{\mu}{\sqrt{1+\sigma^{2}}}, \sqrt{\frac{1+\sigma^{2}(1-\rho)}{1+\sigma^{2}(1+\rho)}}\right) .
$$

Equation (25) follows after subtracting $\langle\phi\rangle^{2}$.

\section{b. Free upcrossing probabilities}

For the free two-point upcrossing probability, we need integrals of the form

$$
I_{n}(a, b)=\int_{0}^{\infty} x^{n} g(x) G(a x+b) d x .
$$

For arbitrary $n$, Eq. (10,01n.4) from Ref. [59] provides the solution

$$
I_{n}(a, b)=\frac{\Gamma((n+1) / 2) 2^{(n-1) / 2}}{\sqrt{2 \pi}} F_{n+1,-b}(\sqrt{n+1} a),
$$

where $F_{v, \mu}(x)$ denotes the cumulative distribution function of noncentral t-distribution with $v$ degrees of freedom and noncentrality parameter $\mu$. Analytical expressions for $F_{v, \mu}(x)$ in terms of $g(x), G(x)$, and $T(h, a)$ can be found in Ref. [91] (the ones in Ref. [59] contain typos). Using these expressions, the solutions for $n=0,1$, and 2 are

$$
\begin{aligned}
& I_{0}(a, b)=\frac{1}{2} G(b B)+T(b B, a), \\
& I_{1}(a, b)=\frac{1}{\sqrt{2 \pi}} G(b)+M_{0}(a, b), \\
& I_{2}(a, b)=I_{0}(a, b)+M_{1}(a, b)
\end{aligned}
$$

where we used the shorthand notation $B=1 / \sqrt{1+a^{2}}$ and

$$
\begin{aligned}
& M_{0}(a, b)=a B g(b B) G(-a b B), \\
& M_{1}(a, b)=B^{2}\left(-a b M_{0}(a, b)+a g(b) / \sqrt{2 \pi}\right) .
\end{aligned}
$$

Since we consider only up to $n=2$, we are spared the increasingly cumbersome expressions for $n>2$.

\section{APPENDIX B: FREE UPCROSSING PROBABILITIES}

The dynamics of the free membrane voltage and the current for the LIF neuron model are given by

$$
\begin{gathered}
\dot{U}=-U+I, \\
\tau_{\mathrm{s}} \dot{I}=-I+\eta,
\end{gathered}
$$

where we measure time in units of the membrane time constant $\tau_{\mathrm{m}}$, i.e., we set $\tau_{\mathrm{m}}=1$. Furthermore, we set $\langle\eta\rangle=0$, i.e., we measure $U$ and $I$ relative to the mean input. Lastly, we define $t=0$ to be the end of the refractory period, i.e., the time when the free dynamics start evolving.

First, we need the distribution of the voltage and the current. Since $\eta$ is a Gaussian process, both are Gaussian for arbitrary time arguments. Thus it is sufficient to calculate the first two conditional cumulants. Throughout, we assume a correlation-free preparation [92], i.e., we assume that $\eta$ and $I$ are uncorrelated prior to $t=0$.

\section{Nonstationary mean and variance of $U$ and $I$}

We need the nonstationary mean and variance of $U$ and $I$ to calculate the free upcrossing probability. For a given initial current and initial voltage, Eqs. (B1) and (B2) lead 
to

$$
\begin{aligned}
& I(t)=I_{0} e^{-t / \tau_{\mathrm{s}}}+\frac{1}{\tau_{\mathrm{s}}} \int_{0}^{t} e^{-(t-s) / \tau_{\mathrm{s}}} \eta(s) d s, \\
& U(t)=U_{0} e^{-t}+\int_{0}^{t} e^{-(t-s)} I(s) d s .
\end{aligned}
$$

This leads immediately to the mean

$$
\begin{aligned}
\mu_{I}(t) & =I_{0} e^{-t / \tau_{\mathrm{s}}}, \\
\mu_{U}(t) & =U_{0} e^{-t}+\frac{\tau_{\mathrm{s}}}{1-\tau_{\mathrm{s}}} I_{0}\left(e^{-t}-e^{-t / \tau_{\mathrm{s}}}\right) .
\end{aligned}
$$

To obtain the variances numerically, we use that they follow linear differential equations: taking the temporal derivatives of $I(t)^{2}, I(t) U(t)$, and $U(t)^{2}$, using Eqs. (B1) and (B2), and averaging leads to

$$
\begin{aligned}
\frac{\tau_{\mathrm{s}}}{2} \dot{\sigma}_{I}^{2} & =-\sigma_{I}^{2}+\sigma_{I \eta}^{2}, \\
\tau_{\mathrm{s}} \dot{\sigma}_{I U}^{2} & =-\left(1+\tau_{\mathrm{s}}\right) \sigma_{I U}^{2}+\tau_{\mathrm{s}} \sigma_{I}^{2}+\sigma_{U \eta}^{2}, \\
\frac{1}{2} \dot{\sigma}_{U}^{2} & =-\sigma_{U}^{2}+\sigma_{I U}^{2} .
\end{aligned}
$$

The initial conditions for all of the above differential equations are $\sigma_{I}^{2}(0)=\sigma_{I U}^{2}(0)=\sigma_{U}^{2}(0)=0$. They are straightforward to solve numerically in the order that they appear, but they require two additional quantities:

$$
\begin{aligned}
\sigma_{I \eta}^{2}(t) & =\frac{1}{\tau_{\mathrm{s}}} \int_{0}^{t} e^{-s / \tau_{\mathrm{s}}} C_{\eta}(s) d s, \\
\sigma_{U \eta}^{2}(t) & =\frac{1}{1-\tau_{\mathrm{s}}} \int_{0}^{t}\left(e^{-s}-e^{-s / \tau_{\mathrm{s}}}\right) C_{\eta}(s) d s,
\end{aligned}
$$

which can be numerically computed using a composite trapezoidal rule. If $C_{\eta}(\tau)$ contains a Dirac delta, $C_{\eta}(\tau)=\hat{C}_{\eta}(\tau)+$ $2 D \delta(\tau)$, we have to separate it analytically in $\sigma_{I \eta}^{2}(t)$ :

$$
\sigma_{I \eta}^{2}(t)=\hat{\sigma}_{I \eta}^{2}(t)+\frac{D}{\tau_{\mathrm{s}}} .
$$

Note the factor $1 / 2$ because we only integrate "half" of the Dirac delta. In $\sigma_{U \eta}^{2}(t)$, the Dirac delta does not contribute because the integrand vanishes at zero, i.e., $\sigma_{U \eta}^{2}(t)=\hat{\sigma}_{U \eta}^{2}(t)$.

Ultimately, we need the cumulants of $U$ and $\dot{U}$ instead of $U$ and $I$. To relate the respective quantities, we use Eq. (B1). For the initial conditions, we have

$$
\dot{U}_{0}=I_{0}-U_{0} \text {. }
$$

The first cumulants are

$$
\begin{aligned}
\mu_{U}(t) & =U_{0} e^{-t}+\left(\dot{U}_{0}+U_{0}\right) A(t), \\
\mu_{\dot{U}}(t) & =-\mu_{U}(t)+\left(\dot{U}_{0}+U_{0}\right) e^{-t / \tau_{\mathrm{s}}} \\
& =-U_{0} e^{-t}+\left(\dot{U}_{0}+U_{0}\right) B(t),
\end{aligned}
$$

where we used Eq. (B1) for $\mu_{\dot{U}}(t)$ and abbreviated

$$
A(t)=\frac{\tau_{\mathrm{s}}}{1-\tau_{\mathrm{s}}}\left(e^{-t}-e^{-t / \tau_{\mathrm{s}}}\right), \quad B(t)=e^{-t / \tau_{\mathrm{s}}}-A(t) .
$$

The second cumulants do not depend on the initial conditions and we get from Eq. (B1):

$$
\begin{aligned}
\sigma_{U \dot{U}}^{2}(t) & =-\sigma_{U}^{2}(t)+\sigma_{I U}^{2}(t), \\
\sigma_{\dot{U}}^{2}(t) & =\sigma_{U}^{2}(t)-2 \sigma_{I U}^{2}(t)+\sigma_{I}^{2}(t) .
\end{aligned}
$$

Finally, we need to marginalize the initial velocity.

We assume that $\dot{U}_{0}$ is Gaussian distributed with mean $\mu_{\dot{U}_{0}}$ and variance $\sigma_{\dot{U}_{0}}^{2}$. Marginalizing $\dot{U}_{0}$ again results in a Gaussian distribution because $p\left(\dot{U}_{0}\right)$ and $p\left(U_{1}, \dot{U}_{1} \mid U_{0}, \dot{U}_{0}\right)$ are Gaussian. Hence, we only need to compute the cumulants. For the mean, we simply have to replace $\dot{U}_{0} \rightarrow \mu_{\dot{U}_{0}}$. The second cumulants are

$$
\begin{gathered}
\tilde{\sigma}_{U}^{2}(t)=\sigma_{U}^{2}(t)+\sigma_{\dot{U}_{0}}^{2} A(t)^{2}, \\
\tilde{\sigma}_{U \dot{U}}^{2}(t)=\sigma_{U \dot{U}}^{2}(t)+\sigma_{\dot{U}_{0}}^{2} A(t) B(t), \\
\tilde{\sigma}_{\dot{U}}^{2}(t)=\sigma_{\dot{U}}^{2}(t)+\sigma_{\dot{U}_{0}}^{2} B(t)^{2} .
\end{gathered}
$$

With this, we can evaluate the mean and the variance numerically from the statistics of $\eta(t)$ and $\dot{U}_{0}$.

\section{Initial velocity distribution}

For the distribution of initial velocities, we assume that the voltage has reached a stationary distribution by the time it crosses the threshold. The velocity at an upcrossing of a stationary Gaussian process is Rayleigh distributed [40]. Because at the threshold we have $\dot{U}_{\text {up }}=-\theta+I_{\text {up }}$ (remember that $t=0$ denotes the end of the refractory period, that the membrane resistance is absorbed into the current, and time is rescaled such that $\tau_{\mathrm{m}}=1$ ), the current is also Rayleigh distributed,

$$
p\left(I_{\text {up }}\right)=\left\{\begin{array}{ll}
\frac{\left(I_{\text {up }}-\theta\right)}{\sigma_{I}^{2}} \exp \left(-\frac{\left(I_{\text {up }}-\theta\right)^{2}}{2 \sigma_{I}^{2}}\right) & \text { for } I_{\text {up }} \geqslant \theta \\
0 & \text { otherwise }
\end{array},\right.
$$

where $\sigma_{I}^{2}=-\ddot{C}_{U}(0)$ with the stationary autocorrelation $C_{U}(\tau)$ of the free voltage. We assume that the further development of the current is also stationary, and neglect the conditional dependencies of the transition probability $p\left(I_{0} \mid I_{\text {up }}\right)$ on the threshold crossing beyond $I_{\text {up }}$, e.g., on $\dot{I}_{\text {up }}$ and $\dot{I}_{\text {up }}$. This transition probability can thus be obtained from the unconstrained ("free") stationary statistics of the current—not conditioned on a threshold crossing — which are Gaussian: $p\left(I_{0} \mid I_{\text {up }}\right)=p_{\text {free }}\left(I_{0}, I_{\text {up }}\right) / p_{\text {free }}\left(I_{\text {up }}\right)$. The unconstrained joint and instantaneous distributions here function as auxiliary quantities for computing $p\left(I_{0} \mid I_{\text {up }}\right)$. The unconstrained joint distribution is a Gaussian with variance $\sigma_{I}^{2}$ and covariance $\sigma_{I}^{2} R_{I}\left(\tau_{\text {ref }}\right)$ where $R_{I}(\tau)=-\ddot{C}_{U}(\tau) / \sigma_{U}^{2}$. We derive $C_{U}(\tau)$ most conveniently by Fourier transforming Eqs. (B1) and (B2), which leads to $S_{U}(f)=S_{\eta}(f) /\left(1+(2 \pi f)^{2}\right) /(1+$ $\left.\left(2 \pi \tau_{\mathrm{s}} f\right)^{2}\right)$, and using the Wiener-Khinchin theorem to obtain the autocorrelation. From the unconstrained joint and instantaneous distributions, we obtain the transition probability $p\left(I_{0} \mid I_{\text {up }}\right)$, which is again a Gaussian with [88]

$$
\tilde{\mu}_{I}\left(\tau_{\mathrm{ref}}\right)=I_{\mathrm{up}} R_{I}\left(\tau_{\mathrm{ref}}\right), \quad \tilde{\sigma}_{I}^{2}\left(\tau_{\mathrm{ref}}\right)=\sigma_{I}^{2}\left(1-R_{I}\left(\tau_{\mathrm{ref}}\right)^{2}\right) .
$$


Combining this with the Rayleigh-distributed $p\left(I_{\text {up }}\right)$ yields

$$
p\left(I_{0}\right)=\int_{\theta}^{\infty} p\left(I_{0} \mid I_{\text {up }}\right) p\left(I_{\text {up }}\right) d I_{\text {up }},
$$

which is not a Gaussian anymore. We only calculate the first two cumulants,

$$
\begin{aligned}
\hat{\mu}_{I}\left(\tau_{\mathrm{ref}}\right) & =\left\langle\tilde{\mu}_{I}\left(\tau_{\mathrm{ref}}\right)\right\rangle_{I_{0}}=\left(\sqrt{\frac{\pi}{2} \sigma_{I}^{2}}+\theta\right) R_{I}\left(\tau_{\mathrm{ref}}\right), \\
\hat{\sigma}_{I}^{2}\left(\tau_{\mathrm{ref}}\right) & =\tilde{\sigma}_{I}^{2}\left(\tau_{\mathrm{ref}}\right)+\left\langle\left(\tilde{\mu}_{I}\left(\tau_{\mathrm{ref}}\right)-\left\langle\tilde{\mu}_{I}\left(\tau_{\mathrm{ref}}\right)\right\rangle_{I_{0}}\right)^{2}\right\rangle_{I_{0}} \\
& =\tilde{\sigma}_{I}^{2}\left(\tau_{\mathrm{ref}}\right)+\frac{4-\pi}{2} \sigma_{I}^{2} R_{I}\left(\tau_{\mathrm{ref}}\right)^{2},
\end{aligned}
$$

and neglect the higher cumulants to arrive at a Gaussian approximation. Finally, after the refractory time we have $\dot{U}_{0}=$ $-V_{\mathrm{r}}+I_{0}$. Combining the above equations leads to

$$
\begin{aligned}
\mu_{\dot{U}_{0}} & =\left(\sqrt{\frac{\pi}{2} \sigma_{I}^{2}}+\theta\right) R_{I}\left(\tau_{\text {ref }}\right)-V_{\mathrm{r}}, \\
\sigma_{\dot{U}_{0}}^{2} & =\sigma_{I}^{2}\left(1-\frac{\pi-2}{2} R_{I}\left(\tau_{\mathrm{ref}}\right)^{2}\right),
\end{aligned}
$$

which determine the Gaussian approximation of the initial velocity distribution.

\section{One-point upcrossing probability}

Here, we calculate the upcrossing probability Eq. (39),

$$
n_{1}(t)=\int_{0}^{\infty} \dot{U}_{1} p\left(\theta, \dot{U}_{1} \mid V_{\mathrm{r}}\right) d \dot{U}_{1}
$$

Due to the linearity of Eqs. (B1) and (B2), the distribution $p\left(\theta, \dot{U}_{1} \mid V_{\mathrm{r}}\right)$ is a Gaussian with the cumulants we calculated above [92]. Hence, it takes the form

$p\left(\theta, \dot{U}_{1} \mid V_{\mathrm{r}}\right)=\frac{1}{\sqrt{\operatorname{det}(2 \pi \mathbf{C})}} \exp \left(-\frac{1}{2}(\mathbf{u}-\boldsymbol{\mu})^{T} \mathbf{C}^{-1}(\mathbf{u}-\boldsymbol{\mu})\right)$,

where $\mathbf{u}^{T}=\left(\theta, \dot{U}_{1}\right)$ and the mean and the correlation matrix are given by

$$
\boldsymbol{\mu}=\left(\begin{array}{c}
\tilde{\mu}_{U}(t) \\
\tilde{\mu}_{\dot{U}}(t)
\end{array}\right), \quad \mathbf{C}=\left(\begin{array}{cc}
\tilde{\sigma}_{U}^{2}(t) & \tilde{\sigma}_{U \dot{U}}^{2}(t) \\
\tilde{\sigma}_{U \dot{U}}^{2}(t) & \tilde{\sigma}_{\dot{U}}^{2}(t)
\end{array}\right) .
$$

Inverting $\mathbf{C}$ leads to

$$
\begin{aligned}
\mathbf{C}^{-1} & =\frac{1}{\operatorname{det}(\mathbf{C})}\left(\begin{array}{cc}
\tilde{\sigma}_{\dot{U}}^{2}(t) & -\tilde{\sigma}_{U \dot{U}}^{2}(t) \\
-\tilde{\sigma}_{U \dot{U}}^{2}(t) & \tilde{\sigma}_{U}^{2}(t)
\end{array}\right), \\
\operatorname{det}(\mathbf{C}) & =\tilde{\sigma}_{U}^{2}(t) \tilde{\sigma}_{\dot{U}}^{2}(t)-\tilde{\sigma}_{U \dot{U}}^{4}(t) .
\end{aligned}
$$

The exponent of $p\left(\theta, \dot{U}_{1} \mid V_{\mathrm{r}}\right)$ takes the form

$$
(\mathbf{u}-\boldsymbol{\mu})^{T} \mathbf{C}^{-1}(\mathbf{u}-\boldsymbol{\mu})=\frac{1}{\operatorname{det}(\mathbf{C})}\left[a \dot{U}_{1}^{2}-2 b \dot{U}_{1}+c^{2}\right]
$$

with $\quad a=\tilde{\sigma}_{U}^{2}(t), \quad b=\tilde{\mu}_{\dot{U}}(t) \tilde{\sigma}_{U}^{2}(t)+\left(\theta-\tilde{\mu}_{U}(t)\right) \tilde{\sigma}_{U \dot{U}}^{2}(t)$ and $c^{2}=\tilde{\mu}_{\dot{U}}(t)^{2} \tilde{\sigma}_{U}^{2}(t)+2\left(\theta-\tilde{\mu}_{U}(t)\right) \tilde{\mu}_{\dot{U}}(t) \tilde{\sigma}_{U \dot{U}}^{2}(t)+(\theta-$ $\left.\tilde{\mu}_{U}(t)\right)^{2} \tilde{\sigma}_{\dot{U}}^{2}(t)$.

Putting it together, $n_{1}$ is given by

$$
n_{1}(t)=\frac{1}{\sqrt{\operatorname{det}(2 \pi \mathbf{C})}} \int_{0}^{\infty} \dot{U}_{1} \exp \left(-\frac{a \dot{U}_{1}^{2}-2 b \dot{U}_{1}+c^{2}}{2 \operatorname{det}(\mathbf{C})}\right) d \dot{U}_{1} \text {. }
$$

The integral can be solved in terms of an error function:

$$
\begin{aligned}
\int_{0}^{\infty} \dot{U}_{1} e^{-\frac{a \dot{U}_{1}^{2}-2 b \dot{U}_{1}+c^{2}}{2 \operatorname{det}(\mathbf{C})}} d \dot{U}= & \frac{\operatorname{det}(\mathbf{C})}{a} e^{-\tilde{c}^{2}} \\
& +\frac{\operatorname{det}(\mathbf{C})}{a} e^{-\tilde{c}^{2}} \sqrt{\pi} \tilde{b} e^{\tilde{b}^{2}}(1+\operatorname{erf}(\tilde{b})),
\end{aligned}
$$

where $\tilde{b}=b / \sqrt{2 a \operatorname{det}(\mathbf{C})}$ and $\tilde{c}=c / \sqrt{2 \operatorname{det}(\mathbf{C})}$. Thus we get

$$
n_{1}(t)=\frac{\sqrt{\operatorname{det}(\mathbf{C})}}{2 \pi \tilde{\sigma}_{U}^{2}(t)} e^{-\tilde{c}^{2}}\left(1+\sqrt{\pi} \tilde{b} e^{\tilde{b}^{2}}(1+\operatorname{erf}(\tilde{b}))\right)
$$

for the free upcrossing rate.

\section{Stationary correlation function of $U$ and $\dot{U}$}

For the stationary two-point upcrossing probability, we need the stationary correlation functions of $U, \dot{U}$, and between $U$ and $\dot{U}$. The power spectrum of $U$ follows from the power spectrum of $\eta$ using

$$
S_{U}(f)=\frac{S_{\eta}(f)}{\left(1+(2 \pi f)^{2}\right)\left(1+\left(2 \pi f \tau_{\mathrm{s}}\right)^{2}\right)} .
$$

An inverse Fourier transform leads to the stationary correlation function $C_{U}(\tau)$. For stationary processes, the formulas

$$
C_{U \dot{U}}(\tau)=-C_{\dot{U} U}(\tau)=\dot{C}_{U}(\tau), \quad C_{\dot{U}}(\tau)=-\ddot{C}_{U}(\tau)
$$

yield the remaining correlation functions. The first formula follows from $\langle U(t) \dot{U}(t+\tau)\rangle=\frac{d}{d \tau}\langle U(t) U(t+\tau)\rangle$ and $\langle\dot{U}(t) U(t+\tau)\rangle=\langle\dot{U}(t-\tau) U(t)\rangle=-\frac{d}{d \tau}\langle U(t-$ $\tau) U(t)\rangle$, the second from $\langle\dot{U}(t) \dot{U}(t+\tau)\rangle=\frac{d}{d \tau}\langle\dot{U}(t) U(t+$ $\tau)\rangle=\frac{d}{d \tau}\langle\dot{U}(t-\tau) U(t)\rangle=-\frac{d^{2}}{d \tau^{2}}\langle U(t-\tau) U(t)\rangle$.

\section{Stationary two-point upcrossing probability}

Here, we calculate the stationary two point upcrossing probability Eq. (40),

$$
n_{2}(\tau)=\int_{0}^{\infty} \int_{0}^{\infty} \dot{U}_{2} \dot{U}_{1} p\left(\theta, \dot{U}_{2} ; \theta, \dot{U}_{1}\right) d \dot{U}_{1} d \dot{U}_{2} .
$$

The joint density $p\left(U_{2}, \dot{U}_{2} ; U_{1}, \dot{U}_{1}\right)$ takes the form

$$
p\left(U_{2}, \dot{U}_{2} ; U_{1}, \dot{U}_{1}\right)=\frac{1}{\sqrt{\operatorname{det}\left(2 \pi \sigma_{U}^{2} \mathbf{C}\right)}} \exp \left(-\frac{1}{2 \sigma_{U}^{2}} \mathbf{u}^{T} \mathbf{C}^{-1} \mathbf{u}\right),
$$

where $\mathbf{u}^{T}=\left(U_{1}, \dot{U}_{1}, U_{2}, \dot{U}_{2}\right)$ and $\sigma_{U}^{2}=C_{U}(0)$. The correlation matrix is given by

$$
\mathbf{C}=\left(\begin{array}{cccc}
1 & 0 & R(\tau) & \dot{R}(\tau) \\
0 & -\ddot{R}(0) & -\dot{R}(\tau) & -\ddot{R}(\tau) \\
R(\tau) & -\dot{R}(\tau) & 1 & 0 \\
\dot{R}(\tau) & -\ddot{R}(\tau) & 0 & -\ddot{R}(0)
\end{array}\right),
$$

where we introduced $C_{U}(\tau)=\sigma_{U}^{2} R(\tau)$ and used $\dot{C}_{U}(0)=0$ for stationary processes with a differentiable correlation function. Inverting $\mathbf{C}$ is cumbersome and eventually leads to

$$
\begin{aligned}
\mathbf{C}^{-1} & =\frac{1}{\operatorname{det}(\mathbf{C})}\left(\begin{array}{cccc}
\alpha & \beta & \gamma & \delta \\
\beta & \epsilon & -\delta & \zeta \\
\gamma & -\delta & \alpha & -\beta \\
\delta & \zeta & -\beta & \epsilon
\end{array}\right) \text { with } \\
\alpha & =\ddot{R}(0)^{2}+\dot{R}(\tau)^{2} \ddot{R}(0)-\ddot{R}(\tau)^{2},
\end{aligned}
$$




$$
\begin{aligned}
\beta & =R(\tau) \dot{R}(\tau) \ddot{R}(0)-\dot{R}(\tau) \ddot{R}(\tau), \\
\gamma & =-R(\tau) \ddot{R}(0)^{2}+R(\tau) \ddot{R}(\tau)^{2}-\dot{R}(\tau)^{2} \ddot{R}(\tau), \\
\delta & =\dot{R}(\tau) \ddot{R}(0)-R(\tau) \dot{R}(\tau) \ddot{R}(\tau)+\dot{R}(\tau)^{3}, \\
\epsilon & =-\ddot{R}(0)+R(\tau)^{2} \ddot{R}(0)-\dot{R}(\tau)^{2}, \\
\zeta & =\ddot{R}(\tau)-R(\tau)^{2} \ddot{R}(\tau)+R(\tau) \dot{R}(\tau)^{2} .
\end{aligned}
$$

The determinant of $\mathbf{C}$ is given by

$$
\begin{aligned}
\operatorname{det}(\mathbf{C})= & {\left[1-R(\tau)^{2}\right]\left[\ddot{R}(0)^{2}-\ddot{R}(\tau)^{2}\right] } \\
& +\dot{R}(\tau)^{2}\left[2 \ddot{R}(0)-2 R(\tau) \ddot{R}(\tau)+\dot{R}(\tau)^{2}\right] .
\end{aligned}
$$

Now, we have to solve the integrals. The exponent of $p\left(U_{2}, \dot{U}_{2} ; U_{1}, \dot{U}_{1}\right)$ takes the form

$$
\begin{aligned}
\mathbf{u}^{T} \mathbf{C}^{-1} \mathbf{u}= & \frac{1}{\operatorname{det}(\mathbf{C})}\left[\epsilon\left(\dot{U}_{1}^{2}+\dot{U}_{2}^{2}\right)+2 \zeta \dot{U}_{1} \dot{U}_{2}\right. \\
& \left.+2(\delta-\beta) \theta\left(\dot{U}_{2}-\dot{U}_{1}\right)+2(\alpha+\gamma) \theta^{2}\right] .
\end{aligned}
$$

With the transformation $v_{1}=\frac{1}{\sqrt{2}}\left(\dot{U}_{2}-\dot{U}_{1}\right)$ and $v_{2}=$ $\frac{1}{\sqrt{2}}\left(\dot{U}_{2}+\dot{U}_{1}\right)$, we have $\dot{U}_{1}^{2}+\dot{U}_{2}^{2}=v_{1}^{2}+v_{2}^{2}, \quad \dot{U}_{1} \dot{U}_{2}=$ $\frac{1}{2}\left(v_{2}^{2}-v_{1}^{2}\right)$ and thus

$$
\begin{aligned}
n_{2}(\tau)= & \frac{e^{-\frac{(\alpha+\gamma) \theta^{2}}{\sigma_{U}^{2} \operatorname{det}(\mathbf{C})}}}{2 \sqrt{\operatorname{det}\left(2 \pi \sigma_{U}^{2} \mathbf{C}\right)}} \int_{0}^{\infty} e^{-\frac{(\epsilon+\zeta) v_{2}^{2}}{2 \sigma_{U}^{2} \operatorname{det}(\mathbf{C})}} \\
& \times \int_{-v_{2}}^{v_{2}}\left(v_{2}^{2}-v_{1}^{2}\right) e^{-\frac{(\epsilon-\zeta) v_{1}^{2}+2 \sqrt{2}(\delta-\beta) \theta v_{1}}{2 \sigma_{U}^{2} \operatorname{det}(\mathbf{C})}} d v_{1} d v_{2} .
\end{aligned}
$$

The substitution $\tilde{v}_{i}=v_{i} / \sqrt{2 \sigma_{U}^{2} \operatorname{det}(\mathbf{C})}$ simplifies the integrals to

$$
\begin{aligned}
n_{2}(\tau)= & \frac{\operatorname{det}(\mathbf{C})^{3 / 2}}{2 \pi^{2}} e^{-\frac{\left((\alpha+\gamma)-\frac{(\beta-\delta)^{2}}{(\epsilon \zeta)}\right) \theta^{2}}{\sigma_{U}^{2} \operatorname{det}(\mathbf{C})}} \int_{0}^{\infty} e^{-(\epsilon+\zeta) \tilde{v}_{2}^{2}} \\
& \times \int_{-\tilde{v}_{2}}^{\tilde{v}_{2}}\left(\tilde{v}_{2}^{2}-\tilde{v}_{1}^{2}\right) e^{-(\epsilon-\zeta)\left(\tilde{v}_{1}-\frac{\beta-\delta}{\epsilon-\zeta} \frac{\theta}{\sqrt{\sigma_{U}^{2} \operatorname{det}(\mathbf{C})}}\right)^{2}} d \tilde{v}_{1} d \tilde{v}_{2} .
\end{aligned}
$$

The inner integrals over $\tilde{v}_{1}$ can be solved in terms of error functions:

$$
\begin{aligned}
I_{0}\left(\tilde{v}_{2} ; a, b\right) \equiv & \int_{-\tilde{v}_{2}}^{\tilde{v}_{2}} e^{-a\left(\tilde{v}_{1}-b\right)^{2}} d \tilde{v}_{1} \\
= & {\left[\frac{1}{2} \sqrt{\frac{\pi}{a}} \operatorname{erf}\left(\tilde{v}_{1}\right)\right]_{\sqrt{a}\left(b-\tilde{v}_{2}\right)}^{\sqrt{a}\left(b+\tilde{v}_{2}\right)}, } \\
I_{1}\left(\tilde{v}_{2} ; a, b\right) \equiv & \int_{-\tilde{v}_{2}}^{\tilde{v}_{2}} \tilde{v}_{1}^{2} e^{-a\left(\tilde{v}_{1}-b\right)^{2}} d x \\
= & {\left[\frac{1+2 a b^{2}}{4 a^{3 / 2}} \sqrt{\pi} \operatorname{erf}\left(\tilde{v}_{1}\right)\right]_{\sqrt{a}\left(b-\tilde{v}_{2}\right)}^{\sqrt{a}\left(b+\tilde{v}_{2}\right)} } \\
& +\left[-\frac{1}{2 a^{3 / 2}} \tilde{v}_{1} e^{-\tilde{v}_{1}^{2}}+\frac{b}{a} e^{-\tilde{v}_{1}^{2}}\right]_{\sqrt{a}\left(b-\tilde{v}_{2}\right)}^{\sqrt{a}\left(b+\tilde{v}_{2}\right)},
\end{aligned}
$$

where $a=\epsilon-\zeta$ and $b=\frac{\beta-\delta}{\epsilon-\zeta} \frac{\theta}{\sqrt{\sigma_{U}^{2} \operatorname{det}(\mathbf{C})}}$. Some of the outer integrals over $\tilde{v}_{2}$ can also be solved in terms of error functions:

$$
\begin{aligned}
& I_{2}(a, b, c) \equiv-\frac{b}{a} \int_{0}^{\infty} e^{-c \tilde{v}_{2}^{2}}\left[e^{-\tilde{v}_{1}^{2}}\right]_{\sqrt{a\left(b-\tilde{v}_{2}\right)}}^{\sqrt{a}\left(b+\tilde{v}_{2}\right)} d \tilde{v}_{2} \\
&= \frac{b}{a} \sqrt{\frac{\pi}{a+c}} e^{-a b^{2}+\frac{a^{2} b^{2}}{a+c}} \operatorname{erf}\left(\frac{a b}{\sqrt{a+c}}\right), \\
& I_{3}(a, b, c) \equiv \frac{1}{2 a^{3 / 2}} \int_{0}^{\infty} e^{-c \tilde{v}_{2}^{2}}\left[\tilde{v}_{1} e^{-\tilde{v}_{1}^{2}}\right]_{\sqrt{a}\left(b-\tilde{v}_{2}\right)}^{\sqrt{a}\left(b+\tilde{v}_{2}\right)} d \tilde{v}_{2} \\
&= \frac{1}{2 a(a+c)} e^{-a b^{2}} \\
&-\frac{b c \sqrt{\frac{\pi}{a+c}}}{2 a(a+c)} e^{-a b^{2}+\frac{a^{2} b^{2}}{a+c}} \operatorname{erf}\left(\frac{a b}{\sqrt{a+c}}\right),
\end{aligned}
$$

with $c=\epsilon+\zeta$. The remaining integrals over $\tilde{v}_{2}$, i.e.

$$
I_{4}(a, b, c) \equiv-\frac{1+2 a b^{2}}{4 a^{3 / 2}} \sqrt{\pi} \int_{0}^{\infty} e^{-c \tilde{v}_{2}^{2}}\left[\operatorname{erf}\left(\tilde{v}_{1}\right)\right]_{\sqrt{a}\left(b-\tilde{v}_{2}\right)}^{\sqrt{a}\left(b+\tilde{v}_{2}\right)} d \tilde{v}_{2},
$$

$I_{5}(a, b, c) \equiv \frac{1}{2} \sqrt{\frac{\pi}{a}} \int_{0}^{\infty} \tilde{v}_{2}^{2} e^{-c \tilde{v}_{2}^{2}}\left[\operatorname{erf}\left(\tilde{v}_{1}\right)\right]_{\sqrt{a}\left(b-\tilde{v}_{2}\right)}^{\sqrt{a}\left(b+\tilde{v}_{2}\right)} d \tilde{v}_{2}$,

can be solved in terms of Owen's $\mathrm{T}$ function $T(h, a)=$ $\frac{1}{2 \pi} \int_{0}^{a} \frac{1}{1+x^{2}} e^{-\frac{1}{2} h^{2}\left(1+x^{2}\right)} d x$ (Ref. [59], see Appendix B 2). Combining everything, we obtain

$$
\begin{aligned}
n_{2}(\tau)= & \frac{\operatorname{det}(\mathbf{C})^{3 / 2}}{(2 \pi)^{2} a c} I_{\mathrm{ana}}(\tilde{a}, \tilde{b}, \tilde{c}, \tilde{d}), \\
I_{\mathrm{ana}}(\tilde{a}, \tilde{b}, \tilde{c}, \tilde{d})= & e^{-\tilde{d}^{2}}+\sqrt{\pi}(1+\tilde{c}) \tilde{b} e^{\tilde{b}^{2}-\tilde{d}^{2}} \operatorname{erf}(\tilde{b}) \\
& +2 \pi \sqrt{\tilde{c}}\left(1 / \tilde{c}-2 \tilde{a}^{2}-1\right) e^{\tilde{a}^{2}-\tilde{d}^{2}} \\
& \times T(\sqrt{2 \tilde{c}} \tilde{b}, 1 / \sqrt{\tilde{c}}),
\end{aligned}
$$

with $\quad \tilde{a}=\sqrt{a} b=\frac{\beta-\delta}{\sqrt{\epsilon-\zeta}} \frac{\theta}{\sqrt{\sigma_{U}^{2} \operatorname{det}(\mathbf{C})}}, \quad \tilde{b}=\frac{a}{\sqrt{a+c}} b=$ $\frac{\beta-\delta}{\sqrt{2 \epsilon}} \frac{\theta}{\sqrt{\sigma_{U}^{2} \operatorname{det}(\mathbf{C})}}, \quad \tilde{c}=\frac{c}{a}=\frac{\epsilon+\zeta}{\epsilon-\zeta}, \tilde{d}=\sqrt{\alpha+\gamma} \frac{\theta}{\sqrt{\sigma_{U}^{2} \operatorname{det}(\mathbf{C})}}$. From $n_{2}(\tau)$, we obtain

$$
Q(\tau)=1-\frac{n_{2}(\tau)}{n_{0}^{2}} \quad \text { and } \quad \eta=2 \int_{0}^{\infty} Q(\tau) d \tau
$$

which allow us to evaluate the Stratonovich approximation.

\section{APPENDIX C: STRATONOVICH APPROXIMATION}

Here, we compare the full Stratonovich approximation Eq. (34),

$$
H_{S}(T)=-\int_{0}^{T} n_{1}(t) \frac{\ln \left(1-\int_{0}^{T} Q\left(t, t^{\prime}\right) n_{1}\left(t^{\prime}\right) d t^{\prime}\right)}{\int_{0}^{T} Q\left(t, t^{\prime}\right) n_{1}\left(t^{\prime}\right) d t^{\prime}} d t,
$$

with its approximation Eq. (35),

$$
h_{S}(t)=\frac{\kappa_{S}}{n_{0}} n_{1}(t), \quad \kappa_{S}=-\frac{1}{\eta} \ln \left(1-n_{0} \eta\right) .
$$

Importantly, both lead to equivalent hazard functions for infinite times [40].

To see this, we need two properties of $n_{1}$ and $n_{2}$. First, the upcrossing probability saturates at a finite value once the 
(a) ISI distribution

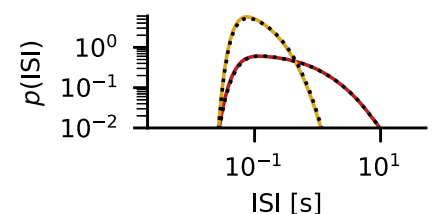

(b)

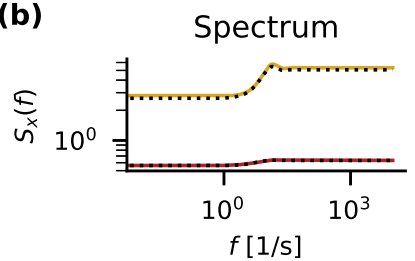

FIG. 12. Comparison of the full Stratonovich approximation with its approximation Eq. (35). (a) ISI distribution from Stratonovich approximation (colors) and Eq. (35) (black). (b) Same for the power spectra. Parameters as in Figs. 8(g) and 8(h).

transient effect of the voltage reset is over, $n_{0}=\lim _{t \rightarrow \infty} n_{1}(t)$. Second, $Q\left(t_{1}, t_{2}\right)=1-\frac{n_{2}\left(t_{1}, t_{2}\right)}{n_{1}\left(t_{1}\right) n_{1}\left(t_{2}\right)}$ decays to zero for $\mid t_{2}-$ $t_{1} \mid \rightarrow \infty$ because the upcrossings decorrelate, $n_{2}\left(t_{1}, t_{2}\right) \rightarrow$ $n_{1}\left(t_{1}\right) n_{1}\left(t_{2}\right)$. Thus one can approximate $\int_{0}^{T} Q\left(t, t^{\prime}\right) n_{1}\left(t^{\prime}\right) d t^{\prime} \approx$ $n_{0} \int_{0}^{\infty} Q\left(t, t^{\prime}\right) d t^{\prime} \equiv n_{0} \eta$ for $0 \ll t \ll T$. Then, neglecting the contributions of $\int_{0}^{T} Q\left(t, t^{\prime}\right) n_{1}\left(t^{\prime}\right) d t^{\prime}-n_{0} \eta$ for $t$ close to 0 or $T$ leads to $H_{S}(T) \approx \int_{0}^{T} \frac{\kappa_{S}}{n_{0}} n_{1}(t) d t$. Neglecting these contributions is justified for large $T$ because the integral is dominated by the contributions in between these boundaries. Hence, we arrive at $\lim _{T \rightarrow \infty} \frac{d}{d T} H_{S}(T)=\lim _{t \rightarrow \infty} h_{S}(t)=\kappa_{S}$ or, in terms of the ISI distribution, $p(T) \sim \exp \left(-\kappa_{S} T\right)$ for $t \rightarrow \infty$.

Since the long-time asymptotics are the same, differences can only occur at short times. In Fig. 12, we compare the full Stratonovich approximation with Eq. (35) for two representative examples. Fortunately, both the resulting ISI distributions [Fig. 12(a)] and the power spectra [Fig. 12(b)] agree closely for all times. Solving the full Stratonovich is numerically challenging (see below); thus, we use the simpler and more efficient approximation throughout in the main text.

\section{Numerics}

Here, we develop a numerical implementation of the Stratonovich approximation that is feasible for long time intervals without excessive demands on the working memory.

For stationary $Q\left(t, t^{\prime}\right)=Q\left(\left|t^{\prime}-t\right|\right)$, the Stratonovich approximation Eq. (34) reads

$$
H_{S}(T)=-\int_{0}^{T} n_{1}(t) \frac{\ln \left[1-\int_{0}^{T} Q\left(\left|t^{\prime}-t\right|\right) n_{1}\left(t^{\prime}\right) d t^{\prime}\right]}{\int_{0}^{T} Q\left(\left|t^{\prime}-t\right|\right) n_{1}\left(t^{\prime}\right) d t^{\prime}} d t .
$$

With the definition

$$
f(T, t)=\int_{0}^{T} Q\left(\left|t^{\prime}-t\right|\right) n_{1}\left(t^{\prime}\right) d t^{\prime},
$$

we have

$$
H_{S}(T)=-\int_{0}^{T} n_{1}(t) \frac{\ln [1-f(T, t)]}{f(T, t)} d t .
$$

Since $Q(\tau \rightarrow \infty) \rightarrow 0$, i.e., it vanishes for long time lags, we can introduce an associated timescale: $Q(\tau) \approx 0$ for all $\tau>$ $\tau_{Q}$. Similarly, $n_{1}(t \rightarrow \infty) \rightarrow n_{0}$ on the timescale $\tau_{n}$ such that $n_{1}(t) \approx n_{0}$ for all $t>\tau_{n}$.

The main problem in computing $H_{S}(T)$ is that a large threedimensional grid is necessary for the three time arguments $t$, $t^{\prime}$, and $T$. To circumvent this problem, we split the domain of integration such that the full grid is only needed in small subdomains. In the remainder of the domain, the integrals can be solved by successive one-dimensional integration.

We consider $f(T, t)$ first. Because $Q\left(\left|t^{\prime}-t\right|\right)$ vanishes for $\left|t^{\prime}-t\right|>\tau_{Q}$, we know that the integrand only contributes in the vicinity of $t$. Thus we can extend the upper limit to infinity, $f(T, t) \approx f(\infty, t)$ if $t<T-\tau_{Q}$. Accordingly, we split the integral where possible:

$$
\begin{aligned}
H_{S}^{T \leqslant \tau_{Q}}(T)= & -\int_{0}^{T} n_{1}(t) \frac{\ln [1-f(T, t)]}{f(T, t)} d t, \\
H_{S}^{T>\tau_{Q}}(T) \approx & -\int_{0}^{T-\tau_{Q}} n_{1}(t) \frac{\ln [1-f(\infty, t)]}{f(\infty, t)} d t \\
& +R^{T>\tau_{Q}}(T), \\
R^{T>\tau_{Q}}(T)= & -\int_{T-\tau_{Q}}^{T} n_{1}(t) \frac{\ln [1-f(T, t)]}{f(T, t)} d t .
\end{aligned}
$$

The remainder $R^{T>\tau_{Q}}(T)$ becomes constant for $T>\tau_{n}+2 \tau_{Q}$ because $n_{1}(t) \approx n_{0}$ in both integrals in this regime and we can set $R^{T>\tau_{n}+2 \tau_{Q}}(T) \approx R^{T>\tau_{n}+2 \tau_{Q}}\left(\tau_{n}+2 \tau_{Q}\right)$. Hence, we only have to calculate the full integral for $H_{S}^{T \leqslant \tau_{Q}}(T)$ and for $R^{T>\tau_{Q}}(T)$ until it is constant.

The remaining integrals in $H_{S}^{T>\tau_{Q}}(T)$ can be solved successively. First, we solve the convolution integral

$$
f(\infty, t)=\int_{0}^{\infty} Q\left(\left|t^{\prime}-t\right|\right) n_{1}\left(t^{\prime}\right) d t^{\prime}
$$

using Fourier transformation. Then, we can insert the result in $H_{S}^{T>\tau_{Q}}(T)$ and solve the integral over $t$. All integrals are approximated by their respective Riemann sum.
[1] A. Bernacchia, H. Seo, D. Lee, and X.-J. Wang, A reservoir of time constants for memory traces in cortical neurons, Nat. Neurosci. 14, 366 (2011).

[2] J. D. Murray, A. Bernacchia, D. J. Freedman, R. Romo, J. D. Wallis, X. Cai, C. Padoa-Schioppa, T. Pasternak, H. Seo, D. Lee et al., A hierarchy of intrinsic timescales across primate cortex, Nat. Neurosci. 17, 1661 (2014).

[3] C. J. Honey, T. Thesen, T. H. Donner, L. J. Silbert, C. E. Carlson, O. Devinsky, W. K. Doyle, N. Rubin, D. J. Heeger, and U. Hasson, Slow cortical dynamics and the accumula- tion of information over long timescales, Neuron 76, 423 (2012).

[4] C. A. Runyan, E. Piasini, S. Panzeri, and C. D. Harvey, Distinct timescales of population coding across cortex, Nature (London) 548, 92 (2017).

[5] T. Ogawa and H. Komatsu, Differential temporal storage capacity in the baseline activity of neurons in macaque frontal eye field and area V4, J. Neurophysiol. 103, 2433 (2010).

[6] M. D. Mauk and D. V. Buonomano, The neural basis of temporal processing, Annu. Rev. Neurosci. 27, 307 (2004). 
[7] R. Rossi-Pool, A. Zainos, M. Alvarez, S. Parra, J. Zizumbo, and R. Romo, Invariant timescale hierarchy across the cortical somatosensory network, Proc. Natl. Acad. Sci. USA 118, e2021843118 (2021).

[8] R. Duarte, A. Seeholzer, K. Zilles, and A. Morrison, Synaptic patterning and the timescales of cortical dynamics, Curr. Opin. Neurobiol. 43, 156 (2017).

[9] X.-J. Wang, Macroscopic gradients of synaptic excitation and inhibition in the neocortex, Nat. Rev. Neurosci. 21, 169 (2020).

[10] R. Chaudhuri, K. Knoblauch, M.-A. Gariel, H. Kennedy, and X.-J. Wang, A large-scale circuit mechanism for hierarchical dynamical processing in the primate cortex, Neuron 88, 419 (2015).

[11] J. M. Huntenburg, P.-L. Bazin, and D. S. Margulies, Large-scale gradients in human cortical organization, Trends Cognit. Sci. 22, 21 (2018).

[12] A. Goulas, K. Zilles, and C. C. Hilgetag, Cortical gradients and laminar projections in mammals, Trends Neurosci. 41, 775 (2018).

[13] S. Marom, Neural timescales or lack thereof, Prog. Neurobiol. 90, 16 (2010).

[14] J. Wilting and V. Priesemann, Between perfectly critical and fully irregular: A reverberating model captures and predicts cortical spike propagation, Cereb. Cortex 29, 2759 (2019).

[15] H. Sompolinsky, A. Crisanti, and H. J. Sommers, Chaos in Random Neural Networks, Phys. Rev. Lett. 61, 259 (1988).

[16] A. Crisanti and H. Sompolinsky, Path integral approach to random neural networks, Phys. Rev. E 98, 062120 (2018).

[17] M. Helias and D. Dahmen, Statistical Field Theory for Neural Networks (Springer, Cham, 2020), Vol. 970, p. 203.

[18] J. Kadmon and H. Sompolinsky, Transition to Chaos in Random Neuronal Networks, Phys. Rev. X 5, 041030 (2015).

[19] C. Huang and B. Doiron, Once upon a (slow) time in the land of recurrent neuronal networks, Curr. Opin. Neurobiol. 46, 31 (2017).

[20] F. Mastrogiuseppe and S. Ostojic, Intrinsically-generated fluctuating activity in excitatory-inhibitory networks, PLOS Comput. Biol. 13, e1005498 (2017).

[21] J. Schuecker, S. Goedeke, and M. Helias, Optimal Sequence Memory in Driven Random Networks, Phys. Rev. X 8, 041029 (2018).

[22] M. Beiran and S. Ostojic, Contrasting the effects of adaptation and synaptic filtering on the timescales of dynamics in recurrent networks, PLOS Comput. Biol. 15, e1006893 (2019).

[23] S. P. Muscinelli, W. Gerstner, and T. Schwalger, How single neuron properties shape chaotic dynamics and signal transmission in random neural networks, PLOS Comput. Biol. 15, e1007122 (2019).

[24] W. Gerstner, W. M. Kistler, R. Naud, and L. Paninski, Neuronal Dynamics. From Single Neurons to Networks and Models of Cognition (Cambridge University Press, Cambridge, 2014).

[25] D. J. Amit and N. Brunel, Model of global spontaneous activity and local structured activity during delay periods in the cerebral cortex, Cereb. Cortex 7, 237 (1997).

[26] N. Brunel, Dynamics of sparsely connected networks of excitatory and inhibitory spiking neurons, J. Comput. Neurosci. 8, 183 (2000).

[27] T. Tetzlaff, M. Helias, G. T. Einevoll, and M. Diesmann, Decorrelation of neural-network activity by inhibitory feedback, PLOS Comput. Biol. 8, e1002596 (2012).
[28] M. Helias, T. Tetzlaff, and M. Diesmann, The correlation structure of local cortical networks intrinsically results from recurrent dynamics, PLOS Comput. Biol. 10, e1003428 (2014).

[29] O. Harish and D. Hansel, Asynchronous rate chaos in spiking neuronal circuits, PLOS Comput. Biol. 11, e1004266 (2015).

[30] C. Keup, T. Kühn, D. Dahmen, and M. Helias, Transient Chaotic Dimensionality Expansion by Recurrent Networks, Phys. Rev. X 11, 021064 (2021).

[31] C. Fulvi Mari, Random Networks of Spiking Neurons: Instability in the Xenopus Tadpole Moto-Neuron Pattern, Phys. Rev. Lett. 85, 210 (2000).

[32] J. Hertz, A. Lerchner, and M. Ahmadi, Mean field methods for cortical dynamics, in Computational Neuroscience: Cortical Dynamics, edited by P. Érdi, A. Esposito, M. Marinaro, and S. Scarpetta (Springer-Verlag Berlin, Heidelberg, 2004), pp. 71-89.

[33] A. Lerchner, G. Sterner, J. Hertz, and M. Ahmadi, Mean field theory for a balanced hypercolumn model of orientation selectivity in primary visual cortex, Network: Comput. Neural Systems 17, 131 (2006).

[34] A. Lerchner, C. Ursta, J. Hertz, M. Ahmadi, P. Ruffiot, and S. Enemark, Response variability in balanced cortical networks, Neural Comput. 18, 634 (2006).

[35] B. Dummer, S. Wieland, and B. Lindner, Self-consistent determination of the spike-train power spectrum in a neural network with sparse connectivity, Front. Comput. Neurosci. 8, 104 (2014).

[36] R. F. Pena, S. Vellmer, D. Bernardi, A. C. Roque, and B. Lindner, Self-consistent scheme for spike-train power spectra in heterogeneous sparse networks, Front. Comput. Neurosci. 12, 9 (2018).

[37] S. Wieland, D. Bernardi, T. Schwalger, and B. Lindner, Slow fluctuations in recurrent networks of spiking neurons, Phys. Rev. E 92, 040901(R) (2015).

[38] R. Zeraati, T. A. Engel, and A. Levina, A flexible Bayesian framework for unbiased estimation of timescales, bioRxiv (2021), doi:10.1101/2020.08.11.245944.

[39] T. Schwalger, M. Deger, and W. Gerstner, Towards a theory of cortical columns: From spiking neurons to interacting neural populations of finite size, PLOS Comput. Biol. 13, e1005507 (2017).

[40] R. L. Stratonovich, Topics in the Theory of Random Noise (Gordon and Breach, New York, 1967).

[41] T. Verechtchaguina, I. M. Sokolov, and L. Schimansky-Geier, First passage time densities in resonate-and-fire models, Phys. Rev. E 73, 031108 (2006).

[42] S. Vellmer and B. Lindner, Theory of spike-train power spectra for multidimensional integrate-and-fire neurons, Phys. Rev. Research 1, 023024 (2019).

[43] T. C. Potjans and M. Diesmann, The cell-type specific cortical microcircuit: Relating structure and activity in a full-scale spiking network model, Cereb. Cortex 24, 785 (2014).

[44] V. Braitenberg and A. Schüz, Cortex: Statistics and Geometry of Neuronal Connectivity, 2nd ed. (Springer-Verlag, Berlin, 1998).

[45] C. van Vreeswijk and H. Sompolinsky, Chaos in neuronal networks with balanced excitatory and inhibitory activity, Science 274, 1724 (1996).

[46] K. D. Harris and A. Thiele, Cortical state and attention, Nat. Rev. Neurosci. 12, 509 (2011). 
[47] R. P. Feynman, A. R. Hibbs, and D. F. Styer, Quantum Mechanics and Path Integrals: Emended Edition (Dover, Mineola, 2010).

[48] G. B. Arous and A. Guionnet, Large deviations for Langevin spin glass dynamics, Probab. Theory Relat. Fields 102, 455 (1995).

[49] A. van Meegen, T. Kühn, and M. Helias, Large-Deviation Approach to Random Recurrent Neuronal Networks: Parameter Inference and Fluctuation-Induced Transitions, Phys. Rev. Lett. 127, 158302 (2021)

[50] A. Roxin, N. Brunel, D. Hansel, G. Mongillo, and C. van Vreeswijk, On the distribution of firing rates in networks of cortical neurons, J. Neurosci. 31, 16217 (2011).

[51] J. Aljadeff, M. Stern, and T. Sharpee, Transition to Chaos in Random Networks with Cell-Type-Specific Connectivity, Phys. Rev. Lett. 114, 088101 (2015).

[52] A. van Meegen and B. Lindner, Self-Consistent Correlations of Randomly Coupled Rotators in the Asynchronous State, Phys. Rev. Lett. 121, 258302 (2018).

[53] T. S. Grigera, Everything you wish to know about correlations but are afraid to ask, arXiv:2002.01750.

[54] R. Gao, R. L. van den Brink, T. Pfeffer, and B. Voytek, Neuronal timescales are functionally dynamic and shaped by cortical microarchitecture, eLife 9, e61277 (2020).

[55] M. Golesorkhi, J. Gomez-Pilar, F. Zilio, N. Berberian, A. Wolff, M. C. Yagoub, and G. Northoff, The brain and its time: intrinsic neural timescales are key for input processing, Commun. Biol. 4, 970 (2021).

[56] J. Wilting and V. Priesemann, Inferring collective dynamical states from widely unobserved systems, Nat. Commun. 9, 2325 (2018).

[57] T. Toyoizumi, K. R. Rad, and L. Paninski, Mean-field approximations for coupled populations of generalized linear model spiking neurons with Markov refractoriness, Neural Comput. 21, 1203 (2009).

[58] M. Krumin and S. Shoham, Generation of spike trains with controlled auto- and cross-correlation functions, Neural Comput. 21, 1642 (2009).

[59] D. B. Owen, A table of normal integrals, Commun. Stat. Simul. Comput. 9, 389 (1980).

[60] W. H. Press, S. A. Teukolsky, W. T. Vetterling, and B. P. Flannery, Numerical Recipes: The Art of Scientific Computing, 3rd ed. (Cambridge University Press, Cambridge, 2007).

[61] T. Fardet, S. B. Vennemo, J. Mitchell, H. Mørk, S. Graber, J. Hahne, S. Spreizer, R. Deepu, G. Trensch, P. Weidel, J. Jordan, J. M. Eppler, D. Terhorst, A. Morrison, C. Linssen, A. Antonietti, K. Dai, A. Serenko, B. Cai, P. Kubaj et al., NEST 2.20.1 (2020), doi:10.5281/zenodo.4018717.

[62] S. Ostojic, Two types of asynchronous activity in networks of excitatory and inhibitory spiking neurons, Nat. Neurosci. 17, 594 (2014)

[63] S. O. Rice, Mathematical analysis of random noise, Bell Syst. Tech. J. 24, 46 (1945), reprinted in [93].

[64] L. M. Ricciardi and S. Sato, A note on first passage time problems for Gaussian processes and varying boundaries, IEEE Trans. Inf. Theory 29, 454 (1983).

[65] T. Schwalger, Mapping input noise to escape noise in integrateand-fire neurons: A level-crossing approach, arXiv:2109.07416 [q-bio.NC].
[66] J.-M. Azaïs and M. Wschebor, Level Sets and Extrema of Random Processes and Fields (Wiley, Hoboken, 2009).

[67] B. Lindner, Interspike interval statistics of neurons driven by colored noise, Phys. Rev. E 69, 022901 (2004).

[68] B. Kriener, H. Enger, T. Tetzlaff, H. E. Plesser, M.-O. Gewaltig, and G. T. Einevoll, Dynamics of self-sustained asynchronousirregular activity in random networks of spiking neurons with strong synapses, Front. Comput. Neurosci. 8, 136 (2014).

[69] E. Ullner, A. Politi, and A. Torcini, Quantitative and qualitative analysis of asynchronous neural activity, Phys. Rev. Research 2, 023103 (2020).

[70] H. Bos, M. Diesmann, and M. Helias, Identifying anatomical origins of coexisting oscillations in the cortical microcircuit, PLOS Comput. Biol. 12, e1005132 (2016).

[71] C. van Vreeswijk and H. Sompolinsky, Chaotic balanced state in a model of cortical circuits, Neural Comput. 10, 1321 (1998).

[72] S. E. Cavanagh, L. T. Hunt, and S. W. Kennerley, A diversity of intrinsic timescales underlie neural computations, Front. Neural Circuits 14, 81 (2020).

[73] D. Dahmen, S. Grün, M. Diesmann, and M. Helias, Second type of criticality in the brain uncovers rich multiple-neuron dynamics, Proc. Nat. Acad. Sci. USA 116, 13051 (2019).

[74] R. Chaudhuri, A. Bernacchia, and X.-J. Wang, A diversity of localized timescales in network activity, eLife 3, e01239 (2014).

[75] A. Litwin-Kumar and B. Doiron, Slow dynamics and high variability in balanced cortical networks with clustered connections, Nat. Neurosci. 15, 1498 (2012).

[76] T. Rost, M. Deger, and M. P. Nawrot, Winnerless competition in clustered balanced networks: Inhibitory assemblies do the trick, Biol. Cybern. 112, 81 (2018).

[77] V. Rostami, T. Rost, A. Riehle, S. J. van Albada, and M. P. Nawrot, Spiking neural network model of motor cortex with joint excitatory and inhibitory clusters reflects task uncertainty, reaction times, and variability dynamics, bioRxiv (2020), doi:10.1101/2020.02.27.968339.

[78] R. Kim and T. J. Sejnowski, Strong inhibitory signaling underlies stable temporal dynamics and working memory in spiking neural networks, Nat. Neurosci. 24, 129 (2021).

[79] G. Deco and V. K. Jirsa, Ongoing cortical activity at rest: Criticality, multistability, and ghost attractors, J. Neurosci. 32, 3366 (2012).

[80] M. Garagnani, G. Lucchese, R. Tomasello, T. Wennekers, and F. Pulvermüller, A spiking neurocomputational model of high-frequency oscillatory brain responses to words and pseudowords, Front. Comput. Neurosci. 10, 145 (2017).

[81] R. Tomasello, M. Garagnani, T. Wennekers, and F Pulvermüller, A neurobiologically constrained cortex model of semantic grounding with spiking neurons and brain-like connectivity, Front. Comput. Neurosci. 12, 88 (2018).

[82] M. Schmidt, R. Bakker, C. C. Hilgetag, M. Diesmann, and S. J. van Albada, Multi-scale account of the network structure of macaque visual cortex, Brain Struct. Func. 223, 1409 (2018).

[83] D. Sussillo, Neural circuits as computational dynamical systems, Curr. Opin. Neurobiol. 25, 156 (2014).

[84] O. Barak, Recurrent neural networks as versatile tools of neuroscience research, Curr. Opin. Neurobiol. 46, 1 (2017).

[85] G. Bellec, D. Salaj, A. Subramoney, R. Legenstein, and W. Maass, Long short-term memory and Learning-to-learn in networks of spiking neurons, in Advances in Neural Information 
Processing Systems 31, edited by S. Bengio, H. Wallach, H. Larochelle, K. Grauman, N. Cesa-Bianchi, and R. Garnett (Curran Associates, Inc., Red Hook, 2018), pp. 795-805.

[86] D. F. Wasmuht, E. Spaak, T. J. Buschman, E. K. Miller, and M. G. Stokes, Intrinsic neuronal dynamics predict distinct functional roles during working memory, Nat. Commun. 9, 3499 (2018).

[87] M. Spitmaan, H. Seo, D. Lee, and A. Soltani, Multiple timescales of neural dynamics and integration of task-relevant signals across cortex, Proc. Nat. Acad. Sci. USA 117, 22522 (2020).

[88] C. K. Williams and C. E. Rasmussen, Gaussian Processes for Machine Learning, 1st ed. (MIT Press, Cambridge, 2006).

[89] P. I. Kuznetsov and R. L. Stratonovich, A note on the mathematical theory of correlated random points, in Non-Linear
Transformations of Stochastic Processes (Pergamon Press, Oxford, 1965), pp. 101-115.

[90] P. Virtanen, R. Gommers, T. E. Oliphant, M. Haberland, T. Reddy, D. Cournapeau, E. Burovski, P. Peterson, W. Weckesser, J. Bright, S. J. van der Walt, M. Brett, J. Wilson, K. J. Millman, N. Mayorov, A. R. J. Nelson, E. Jones, R. Kern, E. Larson, C. J. Carey et al., SciPy 1.0: Fundamental Algorithms for Scientific Computing in Python, Nat. Methods 17, 261 (2020).

[91] D. B. Owen, A special case of a bivariate non-central tdistribution, Biometrika 52, 437 (1965).

[92] P. Hänggi and P. Jung, Colored Noise in Dynamical Systems, in Advances in Chemical Physics 89, edited by I. Prigogine and S. A. Rice (John Wiley \& Sons, Hoboken, 1995), pp. 239- 326.

[93] N. Wax (ed.), Selected Papers on Noise and Stochastic Processes (Dover, New York, 1954). 